\title{
Precise gene models using long-read sequencing reveal a unique poly(A) signal in Giardia lamblia
}

\author{
Danielle Y. Bilodeau ${ }^{1,2}$, Ryan M. Sheridan ${ }^{2}$, Balu Balan $^{3}$, Aaron R. Jex ${ }^{3,4}$, \\ and Olivia S. Rissland ${ }^{1,2}$
}

${ }^{1}$ Department of Biochemistry and Molecular Genetics, University of Colorado Denver School of Medicine, Aurora, CO 80045, USA

${ }^{2}$ RNA BioScience Initiative, University of Colorado Denver School of Medicine, Aurora, CO 80045, USA

${ }^{3}$ Population Health and Immunity Division, The Walter and Eliza Hall Institute of Medical Research, Parkville, Melbourne, VIC, Australia

${ }^{4}$ Faculty of Veterinary and Agricultural Sciences, The University of Melbourne, Parkville, VIC, Australia

*To whom correspondence should be addressed: olivia.rissland@gmail.com 


\begin{abstract}
During pre-mRNA processing, the poly(A) signal is recognized by a protein complex that ensures precise cleavage and polyadenylation of the nascent transcript. The location of this cleavage event establishes the length and sequence of the 3' UTR of an mRNA, thus determining much of its posttranscriptional fate. Using long-read sequencing, we characterize the polyadenylation signal and related sequences surrounding Giardia lamblia cleavage sites for over 2600 genes. We find that G. lamblia uses an AGURAA poly(A) signal, which differs from the mammalian AAUAAA. We also describe how G. lamblia lacks common auxiliary elements found in other eukaryotes, along with the proteins that recognize them. Further, we identify 133 genes with evidence of alternative polyadenylation. These results suggest that despite pared-down cleavage and polyadenylation machinery, $3^{\prime}$ end formation still appears to be an important regulatory step for gene expression in G. lamblia.
\end{abstract}




\section{INTRODUCTION}

Pre-mRNA processing is central to the proper expression and function of a gene. In eukaryotes, pre-mRNA processing involves capping, splicing, and cleavage and polyadenylation, which occur before export to the cytoplasm, and errors at any of these steps can have important consequences for gene expression. During cleavage and polyadenylation, the nascent RNA is cleaved at a precise location, which establishes the $3^{\prime}$ end of the mature transcript, and a poly(A) tail is added, which is required for downstream events in gene expression (Gallie, 1991; Singh et al., 2015). In addition, some genes contain more than one cleavage site, resulting in isoforms with different $3^{\prime}$ UTRs and often different post-transcriptional fates (Mayr \& Bartel, 2009; Sandberg et al., 2008; Tian et al., 2005). Alternative polyadenylation (APA) is widespread in many eukaryotic species, including $S$. cerevisiae, $S$. pombe and plants, and more than half of human and mouse genes have multiple mRNA cleavage sites (Hoque et al., 2013; Liu et al., 2017; Lu et al., 2006; Moqtaderi et al., 2018; Tian et al., 2005; Xing \& Li, 2011). Inappropriate cleavage and polyadenylation can have severe, widespread consequences for gene expression and is associated with cancer and lethality (Whitelaw \& Proudfoot, 1986; Morris et al., 2012; Nourse et al., 2020), highlighting the central importance of this processing step.

Cleavage and polyadenylation is a complex, highly coordinated step that must be highly specific and sensitive. In humans, this process involves 20 core proteins and several cis-acting elements in the mRNA (Kumar et al., 2019). The main sequence element that directs cleavage is the polyadenylation signal (known as the poly(A) signal), which is an AAUAAA hexamer in metazoans (Proudfoot \& Brownlee, 1976; Beaudoing, 2000). This hexamer and variants, such as AUUAAA, are in turn recognized by a multi-protein complex known as the cleavage and polyadenylation specificity factor (or CPSF), which is composed of CPSF160, CPSF30, WDR33, 
CSPF73, CPSF100, Symplekin, and Fip1 (S. Chan et al., 2011; Schönemann et al., 2014). Of these proteins, two (CSPF30 and WDR33) recognize and bind the poly(A) signal and, through other members of the complex, initiate cleavage (S. L. Chan et al., 2014; Clerici et al., 2018; Sun et al., 2018). Although not as clearly defined as in metazoans, A-rich motifs in budding yeast (such as AAGAA) play an analogous role as poly(A) signals (Gross \& Moore, 2001; Hill et al., 2019; Kumar et al., 2019).

In multiple species, the AAUAAA hexamer is insufficient to direct cleavage, and additional auxiliary sequences within the nascent transcript strengthen the poly(A) signal to promote accurate cleavage and polyadenylation (Birse, 1997; Sheets et al., 1990). There are two major auxiliary elements in metazoans: upstream U-rich motifs and downstream U- and UG-rich motifs. The most highly enriched U-rich motif is a UGUA tetramer recognized by proteins in the Cleavage factor $\underline{\operatorname{Im}}$ (CFIm) family (Brown \& Gilmartin, 2003; Venkataraman, 2005). U and GUrich sequences downstream of the cleavage site are recognized by $\underline{\text { Cleavage }} \underline{\text { stimulation factor }}$ proteins (CstF) that also help to strengthen the poly(A) signal and direct the endonuclease CPSF73 for cleavage of the nascent RNA (Hu et al., 2005; Mandel et al., 2006; Sullivan et al., 2009; Takagaki \& Manley, 1997). In yeast, similar auxiliary elements also help define cleavage sites (Baejen et al., 2014; Dichtl, 2002).

Despite our deep knowledge of cleavage and polyadenylation in metazoans and yeast, less is known about the sequences and complexes involved in this process for other eukaryotes. There are over 200,000 species of protists, but we know poly(A) signals for only a handful. For instance, Entamoeba histolytica, which is found in the Amorphea supergroup alongside humans and yeast, uses an AAWUDA poly(A) signal (where $\mathrm{W}$ can be $\mathrm{U}$ or A, and D is any nucleotide but C), reminiscent of the metazoan signal (Hon et al., 2013). Similarly, there has been extensive research 
on pre-mRNA processing in kinetoplastids, such as Trypanosoma and Leishmania (Clayton \& Michaeli, 2011; X.-Q. Li \& Du, 2014). Unlike all other eukaryotes, kinetoplastids transcribe genes as polycistronic mRNAs, which are then cleaved to generate individual transcripts (Campbell et al., 2003; Clayton, 2019). Although trypanosomes contain most of the conserved eukaryotic cleavage and polyadenylation proteins, the cleavage site is established by the trans-splicing of the upstream gene and is not dependent on a specific motif (Clayton, 2013, 2019; Hendriks et al., 2003; Koch et al., 2016). For other protists, the mechanism of cleavage and polyadenylation is less well understood. For instance, a sequencing analysis of Sarcocystis neurona, Neospora caninum, and Toxoplasma gondii was unable to detect a poly(A) signal, although at least in S. neurona, there appears to be alternative polyadenylation during development (Stevens et al., 2018). Plasmodium falciparum, another apicomplexan, also seems to lack a clearly defined poly(A) signal (Oguariri et al., 2006; Siegel et al., 2014). Thus, a substantial amount of eukaryotic diversity remains unexplored for pre-mRNA cleavage and polyadenylation.

One protist that has attracted our interest is Giardia lamblia. A human parasite, G. lamblia is the causative agent of giardiasis, one of the most common intestinal diseases worldwide (Ankarklev et al., 2010). The Giardia clade encompasses multiple species that colonize the intestines of a variety of animals. Within the Giardia clade, G. lamblia is the sole species with the advantage of growing easily in axenic culture (Keister, 1983; Meyer, 1976). Although the exact placement of Giardia species on the eukaryotic tree of life is an ongoing area of investigation (Cacciò \& Ryan, 2008; Monis et al., 2009), it is generally understood to have branched off from traditional model systems, such as Saccharomyces cerevisiae and Drosophila melanogaster, relatively early and has been evolving independently for a long time. Recent phylogenetic analyses place Giardia within the Metamonada supergroup alongside other anaerobic protists like 
Trichomonas, although the term "Excavata" has also been used to describe this supergroup (Burki et al., 2020). Moreover, due to its ease of growth in the lab and its divergence from traditional model systems, G. lamblia presents an opportunity for studying highly conserved processes to see how these compare to what has been previously established.

From the perspective of gene regulation, G. lamblia differs from model organisms in several important ways. First, previous work has suggested that the 3' UTRs of G. lamblia are unusually short, with a median of less than 100 nucleotides (Franzén et al., 2013). This observation has raised fundamental questions about the potential for 3' UTR-mediated post-transcriptional regulation in this organism. Second, consistent with short UTR regions, the genome of G. lamblia is generally very compact such that only eight genes contain introns and five undergo transsplicing, while the number of protein-coding genes is between 5000 to 9000 , depending on genome annotation (Xu, Jex, et al., 2020). Third, G. lamblia has streamlined machinery for transcription (Best, 2004; Morrison et al., 2007), splicing (Iyer et al., 2019; Nixon et al., 2002), and translation (Eiler et al., 2020; L. Li \& Wang, 2004), and lacks many protein components that are essential for viability in most other eukaryotes, such as the translation initiation factor eIF4G (L. Li \& Wang, 2004; Morrison et al., 2007). Finally, G. lamblia exists in two forms, a dormant and hardy cyst and an infectious trophozoite, making it a potential model system to investigate how cell state and developmental transitions affect gene expression. However, despite growing interest in G. lamblia, fundamental aspects of pre-mRNA processing, including the identity of its poly(A) signal, remain unknown.

To provide an initial genome-wide characterization of G. lamblia $3^{\prime}$ end processing, we generated high-quality G. lamblia 3' UTR annotations using two orthogonal high-throughput sequencing methods. Using these data, we identified the G. lamblia poly(A) signal as AGURAA 
(where R indicates a purine). Unlike yeast, G. lamblia uses a specific hexamer as its poly(A) signal. However, this sequence differs from that of metazoans at the second position, using a $\mathrm{G}$ rather than an A. This unusual poly(A) signal has shaped the G. lamblia genome, with the hexamer depleted in coding regions and yet, at times, also overlapping with stop codons to give extremely short 3' UTRs. We found little evidence that known auxiliary sequences play a role in cleavage and polyadenylation, and many of the proteins that would recognize auxiliary sequences seem to also be absent. Together, our results suggest that $G$. lamblia has pared-down pre-mRNA processing machinery or that the sequences and complexes have diverged to the point where they are difficult to identify. Finally, we identified 133 genes with more than one cleavage site. These results increase the number of alternative polyadenylation events in G. lamblia by over 60 -fold (Mok et al., 2005; Que et al., 1996). Our results suggest that, despite simplified cleavage and polyadenylation machinery, $3^{\prime}$ end formation is an important and as-yet underappreciated, mechanism for regulating gene expression in G. lamblia.

\section{RESULTS}

\section{Characterization of G. Iamblia mRNA $3^{\prime}$ ends at nucleotide resolution}

To annotate G. lamblia 3' UTRs, we began with a commercially available 3 '-end sequencing method (QuantSeq), which uses an oligo(dT) primer to sequence 3' ends of polyadenylated RNA with nucleotide resolution. We generated two replicate libraries using trophozoite RNA. Putative cleavage sites were defined by identifying the positions of read peaks downstream of annotated coding regions. Peaks that were within ten nucleotides of each other were merged into a single site. We filtered the sites to only include those where the predicted $3^{\prime}$ UTR showed at least $90 \%$ overlap between biological replicates. In some cases, these libraries led to multiple putative 
cleavage sites per gene, some of which were found tens of thousands of nucleotides away from the nearest open reading frame.

Given the known artefacts of this method (such as internal priming (Adiconis et al., 2013; Nam et al., 2002)) and the challenge of working with a relatively poorly annotated genome, we next used an orthogonal method to validate cleavage sites predicted by the $3^{\prime}$-end seq libraries. We directly sequenced G. lamblia RNA in duplicate using Oxford nanopore technology (ONT) and obtained 1.1 million total reads. ONT sequencing yielded long reads (average length: $940 \mathrm{bp}$ ) that enabled us to unambiguously develop precise gene models and thus enhance the transcriptomic map of G. lamblia.

We used two criteria for ONT read inclusion: reads were required to (1) have a poly(A) tail of at least 30 nucleotides (suggesting that they were derived from mature transcripts, see below) and (2) extend into the open reading frame of the nearest gene (suggesting that they were genuine transcripts from that gene). To validate a cleavage site, we required that it was included in our QuantSeq dataset and had at least one read in either of the two replicate ONT libraries. This method allowed us to remove cleavage sites resulting from internal priming as well as misassigned sites, such as those that belonged to previously unannotated genes (Figure 1A). With this combined approach, we were able to identify 2764 cleavage sites across 2630 genes (which we will refer to as "validated cleavage sites", Figure S1A).

To validate our results, we first compared them to the 3' UTR length of genes that had previously been determined experimentally. For instance, cyst wall protein 1 (CWP1) has been described as having a 36-nucleotide 3' UTR (Hehl et al., 2000), and our measurement gave 37 nucleotides (Table S1). Likewise, we found that NADP-specific glutamate dehydrogenase (GDH) has a 22-nucleotide long 3' UTR (Figure S1B, Table S1), consistent with previous predictions (Yee 
\& Dennis, 1992). Thus, by using a combination of 3 '-end seq and long-read sequencing, we generated a high-confidence dataset of validated cleavage sites for thousands of G. lamblia genes.

We next compared our annotations with those previously predicted on a genome-wide scale (Franzén et al., 2013). The 3' UTR lengths generated by our approach had a median of 59 nucleotides and a similar distribution to previous predictions (Figure 1B). Although these previous estimates and our own annotations were highly correlated (Spearman $r\left[r_{s}\right]=0.95, p<10^{-15}$, Figure 1C), for 693 genes our experimentally determined 3' UTRs were longer than the previous predictions, highlighting the power of our approach. We also observed a significant negative correlation between $3^{\prime}$ UTR length and mRNA expression (Figure 1D, $r_{s}=-0.34, p<10^{-15}$ ), as has been observed in other organisms (Mayr, 2017). This result raises the possibility that 3' UTRs, despite their short length, may carry sufficient regulatory potential to modulate mRNA stability, although the associated mechanisms are unknown.

\section{ONT libraries characterize G. Iamblia poly(A) tails for the first time}

The long-read libraries generated with ONT also allowed us to directly measure poly(A) tails in G. lamblia (Figure S2A). This aspect of RNA biology has been unexplored in G. lamblia, despite it being critical for understanding post-transcriptional regulation and for determining the extent to which standard methods (such as oligo(dT) selection) are appropriate for use in this organism. To examine the reproducibility of our measurements, we first compared the median tail lengths between the two ONT replicates, restricting our analysis to mRNAs with at least ten reads in both replicates. The tail lengths were significantly correlated (Figure S2B, Pearson's $r=0.45, p<10^{-}$ ${ }^{15}$ ), and, even more encouragingly, the median absolute difference in measured tail length between replicates was $8 \mathrm{nt}$, indicating that our tail length measurements were reproducible (Figure 2A). 
To maximise both the resolution and reliability of our results, we next focused on those mRNAs with at least ten reads across both datasets for subsequent analyses (Supplemental Table 1). The median tail length across these transcripts was 69 nucleotides, with $80 \%$ of mRNAs having tails between 58 and $83 \mathrm{nt}$ and $0.2 \%$ having tails shorter than $30 \mathrm{nt}$ (Figure 2B). These lengths are similar to those in Drosophila and human cells, but substantially longer than those in S. cerevisiae (Chang et al., 2014; Krause et al., 2019; Subtelny et al., 2014; Workman et al., 2019; Yu et al., 2020). Interestingly, seven genes showed tails that were reproducibly shorter than 30 nucleotides (Figure S2C-E, Table S2). Of these, four encode ribosomal RNAs, indicating that in G. lamblia structured RNAs are oligoadenylated. In many other eukaryotes, oligo(A) tails are mediated by the TRAMP complex and enable processing and degradation by the nuclear exosome (LaCava et al., 2005). Our data suggest that a similar pathway likely operates in Giardia. From a practical perspective, these tail length measurements indicate that methods using oligo(dT) enrichment steps are suitable for G. lamblia and are unlikely to bias results.

In C. elegans, coverage of poly(A) tails has been sufficiently detailed to show phasing at $\sim 30 \mathrm{nt}$ intervals, consistent with the footprint of poly(A) binding protein on the poly(A) tail of transcripts that are associated with one or multiple copies of poly(A) binding protein (Lima et al., 2017). We therefore examined the ten most highly expressed genes in our dataset to ask whether we could observe something similar, but no phasing was observed. The overall distribution of reads remained constant when looking individually at highly expressed genes (Figure 2C-D), and we also saw no evidence of phasing when looking across all genes (Figure S2A).

Previous work in yeast, humans, and other eukaryotes (Lima et al., 2017; Rissland, 2017; Subtelny et al., 2014) has shown that mRNAs encoding ribosomal proteins (r-proteins) have some of the shortest poly(A) tails in the transcriptome, and we next asked whether this trend held in $G$. 
lamblia. As in other eukaryotes, r-protein mRNAs had significantly shorter poly(A) tails than those on other mRNAs (median 56.4 vs 69 nucleotides, respectively; Mann-Whitney $\mathrm{U}$ test, $p<10^{-15}$; Figure 2E). To ask what biological processes were associated with short or long poly(A) tails, we determined GO enrichment in the genes whose mRNAs were in bins for the $30 \%$ shortest or longest median tail length (Figure 2F) (Ashburner et al., 2000; The Gene Ontology Consortium et al., 2021). Although the poor annotation of the G. lamblia genome and the abundance of hypothetical proteins can make these types of analyses challenging, several processes such as nucleotide metabolism and organophosphate biosynthesis were enriched in genes whose mRNAs had long poly(A) tails, while those having short tails were enriched for several other processes, including ribosome assembly, cytoplasmic translation, rRNA maturation and protein folding. Taken together, these data indicate the underlying mechanisms leading to highly expressed mRNAs, like those encoding r-proteins, are conserved in G. lamblia despite its pared-down molecular machinery.

\section{G. lamblia uses an unusual poly(A) signal}

From our list of validated cleavage sites, we next asked which poly(A) signals, if any, G. lamblia uses. As a first approach, we looked at the frequency of each nucleotide in a 60-nucleotide window centered on the validated cleavage sites (Figure 3A). We noticed A-richness approximately 10 nucleotides upstream of the cleavage site, as well as a distinct A-peak directly downstream. There was also an enrichment of $U$ nucleotides both up and downstream of this region, similar to that seen in other organisms (Tian et al., 2005; Tian \& Graber, 2012), Figure S3A). These results suggest that $G$. lamblia has sequence preferences for defining cleavage sites. 
We next wanted to define the precise poly(A) signal used in G. lamblia. To do so, we focused on genes with only one validated cleavage site and counted the occurrences of hexameric motifs previously identified in humans (Beaudoing, 2000). When we performed this analysis on human RefSeq transcript annotations, AAUAAA was the most abundant polyadenylation signal, as expected (Figure 3B). In contrast, distinct but related motifs were the most highly enriched in our G. lamblia dataset: AGUAAA and AGUGAA were found in $45 \%$ and $15 \%$ of genes, respectively. In contrast, AAUAAA was used more rarely and occurred in only 5\% of genes.

As an independent approach, we searched for hexameric motifs occurring within the first 30 nucleotides upstream of human and G. lamblia cleavage sites using the MEME package (Bailey et al., 2009). This unbiased approach confirmed the strong enrichment for the G nucleotide at position 2 of the G. lamblia poly(A) signal and the strong preference for a purine at position 4 (Figure 3C). Our identified poly(A) signal is also consistent with early studies of individual $G$. lamblia genes that suggested an AGURAA motif as the polyadenylation signal (Peattie et al., 1989; Que et al., 1996; Yee \& Dennis, 1992) — an observation we have now confirmed on a genomewide scale.

Interestingly, although metazoan poly(A) signals are usually found 10 to 30 nucleotides upstream of the cleavage site (Kumar et al., 2019; Figure S3B), G. lamblia signals tended to be closer to the cleavage site (Figure 3D). In over 90\% of genes with an AGUAAA signal, the motif was less than 20 nucleotides from the cleavage site, and the most common distance was 13-15 nucleotides, an observation consistent with the general compactness of the G. lamblia genome.

\section{Implications of unusual poly(A) signal on the G. lamblia genome}


We next wished to investigate how the unusual poly(A) signal has shaped the G. lamblia genome. First, given that AGUAAA and AGUGAA are poly(A) signals, we would expect them to be depleted in open reading frames as their presence could lead to premature cleavage. To test this prediction, we counted the occurrence of both motifs and compared them to the frequency of their shuffled sequences (e.g., AAUAGA). We found that AGUAAA is strongly depleted in open reading frames compared to the shuffled sequences, while the depletion of AGUGAA was more modest, consistent with the prediction that AGUAAA is the preferred signal (Figure 4A, B).

We then investigated the relationship between poly(A) signals and stop codons. A recent study of Giardia muris that reported that many genes have an overlap between these signals $(\mathrm{Xu}$, Jiménez-González, et al., 2020), and genomic analysis of Spironucleus salmonicida, another diplomonad, has likewise indicated a strong "dual use" of poly(A) signals as stop codons (Xu et al., 2014). In the case of $S$. salmonicida, the stop codon (UGA) is predominantly used throughout the genome, overlapping with a predicted an AGUGA poly(A) signal (Xu et al., 2014). Given the short length of 3' UTRs in G. lamblia, we wondered whether this overlap of signals might also occur here. We first calculated the frequency of each stop codon across all open reading frames. We did not observe a strong preference for any stop codon, and UAA (which would allow for an AG-UAA motif) was the least abundant of the three stop codons (Figure 4C). We next looked more closely at the nucleotides preceding the stop codon and asked whether there was a preference for AA, AU, AC, or AG. Of these, only a (N)AG sequence in front of the stop codon will allow

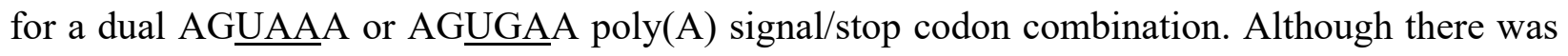
no enrichment for the UAA stop codon itself, it was much more likely to be preceded by an NAG codon than the other codons. We also observed a preference for AG dinucleotides preceding UGA, and a more modest enrichment for UAG, which would not support a dual-use poly(A) signal/stop 
codon (Figure 4D). In contrast, AA dinucleotides showed no such preference, providing an additional line of support that $G$. lamblia does not use the AAUAAA hexamer.

Two alternative models could explain the nucleotide bias in the codon preceding the stop codon: the first is that NAG-UAA and NAG-UGA represent genuine poly(A) signals and the second is that their presence is simply a consequence of codon usage or amino acid preferences. To distinguish between these possibilities, we compared the expected and observed frequencies of NAG sequences preceding the stop codon. Consistent with NAG-UAA serving as a dual poly(A) signal/stop codon, this pair occurred more frequently than expected based on the frequencies of either alone. The same was not true for NAG-UGA (Figure 4E). To investigate this issue further, we examined the 3' UTR lengths of genes with the potential dual use AG-UAA or AG-UGA stop codons. Compared with other genes, 3' UTR lengths were shorter for both AGUAA- and AGUGAending transcripts $\left(p<10^{-15}\right.$ and $p<10^{-12}$, respectively; Figure 4F). In the case of AGUAA, the median length was 11 nucleotides, which is in the window of distances between genuine poly(A) signals and cleavage sites. These analyses indicate that NAG-URA sequences can act as genuine dual-use stop codons and poly(A) signals. In other words, in G. lamblia, stop codons have acquired the ability to also act as poly(A) signals for $\sim 15 \%$ of genes. This dual usage has not reached the levels predicted in G. muris and S. salmonicida, suggesting that this aspect of genome organization is evolving relatively rapidly within the diplomonad order.

\section{Eukaryotic auxiliary elements are poorly enriched around G. Iamblia cleavage sites}

We have an advanced understanding of the sequences and proteins involved in recognition of polyadenylation signals and auxiliary elements in other eukaryotes. In metazoans, there are three main complexes that recognize the polyadenylation signal, upstream U-rich motifs and 
downstream U- and UG-rich motifs: CPSF, CFIm and CstF complexes, respectively (Brown \& Gilmartin, 2003; Kumar et al., 2019; Takagaki \& Manley, 1997). However, it is completely unknown whether G. lamblia also makes use of auxiliary elements to define cleavage sites.

To investigate whether these sequences were conserved in G. lamblia, we began by searching for orthologs to the associated proteins. Although we readily identified candidates for the CPSF complex (which recognizes the poly(A) signal), we found only low-confidence candidates for members of the CstF complex (which recognizes downstream U-rich motifs), and we were unable to identify orthologs for the CFlm proteins (which recognize upstream U-rich motifs and UGUA; Figure 5A, Table S2).

We next examined the sequences surrounding G. lamblia cleavage sites to investigate the extent to which the corresponding recognition sequences of these complexes were enriched. We interrogated sequences 20 to 50 nucleotides upstream of the cleavage sites where the highly conserved UGUA motif is found in other eukaryotes (Brown \& Gilmartin, 2003; Millevoi \& Vagner, 2010). By counting the number of occurrences of UGUA as well as shuffled versions of the motif, we observed a strong preference for UGUA in the human genome, as expected. In contrast, we saw only a slight enrichment in G. lamblia (Figure 5B). Consistent with this result, when we performed an unbiased motif search using MEME, no sequences were enriched in this region (data not shown). This poor sequence conservation, combined with our inability to identify any CFlm orthologs, suggest that upstream motifs either do not play a role in the processing of $G$. lamblia transcripts or are sufficiently divergent as to preclude identification.

Next, we searched for downstream auxiliary elements. In other organisms, these downstream elements lack a consensus motif, but rather are generally U-rich. Thus, we looked for hexamers that were enriched around strong poly(A) sites in human sequences (Hu et al., 2005). As 
expected, we found that these sequences were highly enriched in regions downstream of cleavage sites in humans, but almost completely absent upstream. In contrast, in G. lamblia the sequences were equally present either side of cleavage sites (Figure 5C), which suggests that G. lamblia does not use conserved downstream auxiliary elements. However, because we observed a strong U bias downstream of the cleavage site in metagene analyses (Figure 3A) and the ambiguous presence of putative CstF orthologs raise the possibility that instead divergent cis-elements and proteins may help define genuine cleavage sites, we turned to an unbiased approach to look for enriched motifs. For each gene containing a single cleavage site and an AGUAAA poly(A) signal, we searched for all possible 6-nucleotide motifs in the 50 nucleotides upstream and downstream of the signal. We found an enrichment for A-rich and AU-rich motifs in the downstream regions, and a depletion of more canonical GU-rich motifs (Figure 5D). These results support our observation that any sequences that may help strengthen poly(A) signals in G. lamblia have diverged substantially from those found in classical model eukaryotes.

\section{Evidence of alternative polyadenylation in G. Iamblia}

There are two previously described examples of alternative polyadenylation in the G. lamblia literature (Einarsson et al., 2016; Mok et al., 2005; Que et al., 1996), and so alternative polyadenylation has not been believed to be widespread. However, as mentioned above, when annotating cleavage sites, we unexpectedly found 133 genes showing evidence of alternative polyadenylation (Figure 6A, Table S1), suggesting that alternative polyadenylation may be more common in G. lamblia than previously suspected (Figure SA).

The majority of these alternative cleavage sites were within 100 nucleotides of each other, although for 20 genes the distal cleavage site was over 1000 nucleotides downstream from the 
proximal one (Figure 6B). Nonetheless, given the short length of $3^{\prime}$ UTRs in G. lamblia, in 53\% of cases, usage of the distal site more than doubled the amount of regulatory sequence (Figure 6C, Figure S4B). Interestingly, even usage of the proximal site resulted in longer 3' UTRs than in the rest of the transcriptome (Figure 6D: 135 nucleotides vs 59 nucleotides, $p=0.0052$ ). In humans, proximal sites often use "weaker" poly(A) signals than distal sites (Hu et al., 2005; Legendre \& Gautheret, 2003), and so we looked at poly(A) signals for these examples in G. lamblia. We found that distal cleavage sites are more likely to use AGUAAA and that proximal sites have a higher frequency of alternate signals such as AGUGAA, which is consistent with a preference for AGUAAA over AGUGAA in the transcriptome (Figure 6E).

The presence of alternative poly(A) sites, as well as the generally longer 3' UTR lengths observed, suggested that the regulation of this subset of genes may be biologically important. We observed a slight difference in overall expression between genes that had a single or multiple cleavage sites (median FPKM: 86 and 59.2, respectively; $p=0.00024$; Figure S4C), although there was no difference in poly(A)-tail lengths $(p=0.39$; Figure S4D). We also performed a gene ontology enrichment analysis, but no significant processes were enriched in genes undergoing alternative polyadenylation. We suspect that this result may be because more than $50 \%$ of genes are uncharacterized in G. lamblia, which limits the power of these approaches. Indeed, 12 of the alternative polyadenylation genes are described as 'putative', and 72 are hypothetical proteins or unspecified products. Nonetheless, two ribosomal protein genes (S4 and S28), as well as nine predicted kinases use alternative polyadenylation (Supplemental Table 3), raising the intriguing possibility that alternative polyadenylation may be important for the G. lamblia life cycle.

\section{DISCUSSION}


Here, we empirically annotated the 3' UTRs for 2630 expressed genes in G. lamblia using a combination of 3'-end short- and long-read sequencing. According to our RNA-Seq data (Eiler et al., 2020), 6616 of the 9700 predicted coding genes in the genome annotation used for this study are expressed at an FPKM of 10 or higher. This indicates that we have annotated about $40 \%$ of the expressed transcriptome. Although one barrier to annotating the rest of the genome is low ONT sequencing depth (relative to short-read based sequencing) and the very low RNA expression of the remaining genes (average FPKM $=1.89$ ), direct long-read RNA sequencing was nonetheless instrumental in overcoming some of the difficulties associated with the study of an organism whose genome remains relatively unannotated compared to traditional model systems. Critically, our use of ONT sequencing mitigated known issues with $3^{\prime}$ end short read sequences (Adiconis et al., 2013) and directly linked cleavage sites and open reading frames.

Our work confirms the early putative hypothesis for the G. lamblia poly(A) signal (Peattie et al., 1989; Que et al., 1996; Yee \& Dennis, 1992) and demonstrates that G. lamblia uses AGURAA on a genome-wide scale. Interestingly, the most frequent signal (AGUAAA) differs from the metazoan AAUAAA motif by only a single nucleotide, using a $\mathrm{G}$ at position 2 rather than an A-but the two most common G. lamblia signals (AGURAA) are used only rarely in metazoans (Hu et al., 2005). An interesting future question is how this divergent sequence is recognized. In metazoans, the poly(A) signal is recognized by CPSF30 and WDR33 (Casañal et al., 2017; S. L. Chan et al., 2014; Clerici et al., 2018). We were able to identify putative orthologs to these key players, but orthologs for supporting proteins such as CPSF-100 and Symplekin remain to be found (Table S3). The predicted CPSF30 ortholog in G. lamblia is similar to the human protein but contains four zinc finger (ZF) motifs instead of five, corresponding to motifs 2-4 in human CPSF30. Binding between CPSF30 and the AAUAAA motif is mediated by ZF2 and ZF3, 
suggesting that the core elements of poly(A) signal recognition are likely conserved in G. lamblia (Barabino et al., 2000; Kumar et al., 2019; Schönemann et al., 2014). Furthermore, the highly conserved residues on CPSF30 that are critical for recognition of the motif appear to be conserved and do not offer immediate insight into why G. lamblia uses a different signal. Identifying the appropriate orthologs and their sequence, structure, and biochemical preferences will be an important next step for understanding the basis of the unique G. lamblia poly(A) signal and its evolution.

Although starting with conserved eukaryotic sequences proved to be a good strategy when looking for polyadenylation signals, it was not the case for auxiliary elements. We were unable to find evidence of enrichment for any of the most common metazoan sequences that are found up or downstream of cleavage sites. It is therefore likely that any motifs outside the poly(A) signal used by G. lamblia to direct $3^{\prime}$-end processing have diverged significantly from those found in other eukaryotes, and their identification will likely require additional functional studies.

Finally, an unexpected finding from our study of 3' UTRs is that 133 genes use alternative polyadenylation. Previous reports had identified only two cases (Mok et al., 2005; Que et al., 1996), a result that had led to a view that alternative polyadenylation was as rare as splicing in $G$. lamblia. Our results demonstrate that, contrary to this model, alternative polyadenylation is a more generally used mechanism, adding to the regulatory layers used by G. lamblia. Indeed, our results raise more intriguing questions about how cleavage and polyadenylation is regulated. For instance, how do these different $3^{\prime}$ UTR isoforms affect transcript stability and translation? Why do some genes use alternative polyadenylation and not others? Previous reports have suggested that encystation impacts gene expression as well as cleavage and polyadenylation of individual genes (Einarsson et al., 2016; Mok et al., 2005; Que et al., 1996). An intriguing possibility is that 
alternative polyadenylation may be especially important during this process or in the cyst itself (which is transcriptionally silent), and it will be exciting to explore this and other questions in the future.

\section{MATERIALS AND METHODS}

\section{Trophozoite culture and RNA extraction}

G. lamblia trophozoites (assemblage A, strain WB clone C6) were grown in modified TYS-33 media as per standard protocols (Keister, 1983). Cells were harvested by placing culture tubes on ice for 10 minutes, then spun down for 5 minutes at $800 \mathrm{x} \mathrm{g}$ at $4{ }^{\circ} \mathrm{C}$. Cell pellets were washed twice in 1xPBS. RNA was extracted from trophozoite pellets with hot acid phenol as previously described (Collart \& Oliviero, 1993).

\section{RNA sequencing and analysis}

Previously generated RNASeq libraries used in this study are available from the GEO (GSE158187). 3'-end libraries were generated with the QuantSeq 3' mRNA-Seq Library REV kit from Lexogen (catalog \#016) according to the manufacturer's protocol. Libraries were sequenced at the Genomics and Microarray Shared Resource at the University of Colorado Denver Cancer Center. All sequencing data generated in this study are available from the GEO, accession number GSE168675.

Nanopore libraries were prepared according to the Direct RNA sequencing protocol from ONT (SQK-RNA002). Because the lengths of poly(A) tails were unknown when we initiated this study, total RNA was used in place of poly(A) selected RNA. Libraries were sequenced on a FLO- 
MIN106 flow cell and minION sequencing device. Base-calling was completed by the MinKNOW software (Nanopore) on default settings.

Adaptors were trimmed from 3'-end reads using Cutadapt v2.3. RNASeq and QuantSeq libraries were aligned using STAR 2.5.2a (Dobin et al., 2013). Nanopore libraries were aligned with minimap2 version 2.17-r974-dirty (H. Li, 2018). All libraries were mapped to the Giardia lamblia WBC6 genome version 50 downloaded from the GiardiaDB website on February 8, 2021 (https://giardiadb.org). Poly(A)-tail lengths from the Nanopore libraries were measured using Nanopolish version 0.11.1 (Loman et al., 2015). Mapped nanopore reads were assigned to their corresponding gene using featureCounts version 2.0.0 (Liao et al., 2014).

\section{Identification and validation of cleavage sites}

3' UTRs were annotated by first identifying poly(A) sites. Poly(A) sites were mapped by identifying peaks of poly(A) reads that aligned downstream of coding regions but did not overlap the following gene. Potential poly(A) sites were filtered to only include those that have at least ten reads. Sites that were within 10 nucleotides of each other were combined into a single peak with coordinates representing the center point between the sites.

For each putative cleavage site, a list of coordinates was generated that went 10 nucleotides up and downstream from the site. For each gene, ONT reads with $3^{\prime}$ ends that ended within the corresponding window were selected. Reads were then further filtered to keep only those that contained a poly(A) tail of at least 30 nucleotides and for which the $5^{\prime}$ end of the read fell within the open reading frame of the associated gene. Sites with at least one read from either replicate of the ONT libraries that satisfied all conditions were kept as validated sites. Analyses and plotting 
were performed in $\mathrm{R}$ version 4.0.3 and Python version 3.8.3 from in-house scripts. All genome browser images were generated with IGV version 2.8.10.

\section{Unbiased motif analysis}

Motif-based sequence analysis was done using the MEME suite software at meme-suite.org (Bailey et al., 2009). We searched for a maximum of 3 motifs on the given strand only with minimum and maximum motif lengths of 6 and 50 nucleotides, respectively.

\section{Ortholog identification}

Human protein sequences were used to search for orthologs in G. lamblia by BLAST search. Where it was difficult to identify the most likely ortholog among the search results, the yeast protein sequence was used for a complimentary search. Searches were conducted on giardiadb.org. For CPSF160 and WDR33, human proteins containing similar domains were used to perform a multiple sequence alignment, which was then used to generate a Hidden Markov Model. We then initiated a search across the G. lamblia proteome in search of proteins that have a similar domain and sequence.

\section{ACKNOWLEDGEMENTS}

We thank Dr. Lori Passmore and Vytaute Boreikaite for insightful discussions about the CPSF complex. We thank members of the Rissland, Jagannathan, Bentley, Mukherjee, and Taliaferro labs for helpful and thoughtful discussions during our weekly lab meetings. We thank Prof. Staffan Svärd for assistance with G. lamblia. We are grateful to Dr. Passmore, Dr. Bentley, Dr. Mukherjee, and Dr. Ramachandran for their feedback on this manuscript. This work was supported by NIH 
grants R35GM128680 (OSR) and the RNA Bioscience Initiative. ARJ is supported by the Australian National Health and Medical Research Council L1 Investigator grant (APP1194330) and the Walter and Eliza Hall Institute of Medical Research receives support through the Victorian State Government Operational Infrastructure Support and Australian Government National Health and Medical Research Council Independent Research Institute Infrastructure Support Scheme. RMS is supported by T32 AI074491.

\section{FIGURE LEGENDS}

Figure 1. Characterization of G. Iamblia $3^{\prime}$ ends at nucleotide resolution. (A) Genome browser image looking at the $3^{\prime}$ end of GL50803_104139 and displaying coverage of ONT libraries (top) and $3^{\prime}$-end libraries (bottom). Of the two cleavage sites predicted by the 3 '-end libraries, one is supported by the ONT libraries (green box), while the other appears to belong to a previously unannotated transcript (orange box). (B) Distribution of 3' UTR lengths in previously published work ((Franzén et al., 2013), left) and this study (right). (C) Hexagonal heatmap comparing published estimates of $3^{\prime}$ UTR lengths (x-axis) and the new dataset from this study (y-axis). (D) 3' UTR length is negatively correlated with expression. Shown is a hexagonal heatmap comparing 3' UTR length (this study) and mRNA expression in Fragments Per Kilobase of transcript per Million mapped reads (FPKM, from accession number GSE158187).

Figure 2. Poly(A)-tail measurements provide new insights. (A) Violin plot showing the absolute difference in poly(A)-tail measurements between ONT replicates. (B) Distribution of median poly(A)-tail length across both ONT replicates. Only mRNAs with a combined minimum of 10 reads are included. Median is 69nt. (C) Distribution of poly(A)-tail lengths for reads aligning to 
GL50803_40591. (D) As in C but for GL50803_10311. (E) Comparison of poly(A)-tail length between mRNAs encoding ribosomal proteins (median 56.4nt) and all other mRNAs (median 69.0nt). Only genes with a minimum of 10 ONT reads were selected for this analysis. (F) GEO enrichment terms for genes with short (orange) or long (blue) poly(A) tails. Only genes with a minimum of 10 ONT reads were selected for this analysis.

Figure 3. G. Iamblia uses an unusual poly(A) signal. (A) Nucleotide frequency in the 60nucleotide window centered on all 2860 validated cleavage sites from this study. (B) Frequency of common poly(A) signals identified in studies of human transcripts (Beaudoing, 2000). Sequences 30 nucleotides upstream of cleavage sites from the human RefSeq annotations and validated $G$. lamblia sites from this study were used to search for common motifs. Plotted is the frequency of each signal in human (left) and G. lamblia (right). (C) MEME analysis of upstream sequences. The same sequences as in (B) were uploaded to the meme-suite and a search was conducted for enriched hexamers. Shown is the top motif for human (left) and G. lamblia (right). (D) For all validated cleavage sites containing an AGUAAA motif in the last 40 nucleotides of the mRNA, this bar graph shows the distance between the motif and the end of the read. Distances are counted from the first A of the motif.

Figure 4. Implications of unusual poly(A) signal on G. Iamblia open reading frames. (A) Open reading frames are depleted for G. lamblia's poly(A) signal. Open reading frame sequences were used to count the occurrence of AGUAAA vs all shuffled versions of the motif. (B) As in A, but with the AGUGAA poly(A) signal. (C) Frequency of stop codons across all annotated G. lamblia open reading frames. (D) Nucleotides preceding a stop are enriched for AG over other AN 
dinucleotides. For each stop codon, this bar graph shows how many were preceded by the different AN dinucleotide sequences. (E) As in D, but comparing expected vs observed frequencies. The expected frequency for each sequence context was calculated from the total frequency of each codon across all open reading frames. (F) Distribution of 3' UTR lengths for genes where there is no overlap of poly(A) signal and stop codon (left), genes where there is an AG dinucleotide preceding a UAA stop codon (middle), and genes where there is an AG preceding a UGA stop codon (right).

Figure 5. Conserved auxiliary elements are poorly enriched around G. lamblia cleavage sites. (A) Conserved pre-mRNA processing proteins and the sequences they recognize. The left panel shows the location and motifs of key sequences found around human cleavage sites. Right panel shows the human orthologs of core processing complexes for the recognition of poly(A) signals and surrounding sequences. Dots indicate whether an ortholog was readily identifiable in $G$. lamblia (black circle), whether ortholog identification was ambiguous (grey circle), or whether no orthologs were found (white circle). (B) The conserved UGUA motif is not enriched upstream of G. lamblia cleavage sites. Sequences 20 to 50 nucleotides upstream of cleavage sites were used to count the frequency of UGUA or shuffled versions of the motif. Plotted is the number of times each motif was found in human (left) and G. lamblia (right) sequences. (C) GU-rich elements are not enriched downstream of $G$. lamblia cleavage sites. Sequences 40 nucleotides up and downstream of human and G. lamblia cleavage sites were used to count the occurrence of U- and GU-rich motifs enriched downstream of strong human cleavage sites ( $\mathrm{Hu}$ et al., 2005). Plotted is the frequency of each motif upstream (grey) or downstream (green) of human (left) and G. lamblia (right) cleavage sites. (D) MA plot of enriched and depleted 6-mer sequences around 
polyadenylation signals. All single cleavage site genes from our dataset that contain an AGUAAA were selected for this analysis. Sequences 50 nucleotides upstream and downstream of the signal were used to search for all possible 6-nucleotide motifs. Plotted is the average count of each motif versus its enrichment in downstream sequences. Red dots are motifs that showed at least a 4-fold enrichment or depletion in downstream regions and with an average count of at least 15 occurrences.

Figure 6. Evidence of alternative polyadenylation in G. Iamblia. (A) Genome browser image looking at the 3' end of GL50803_5772 and displaying coverage of ONT libraries (top) and 3'-end libraries (bottom). Both methods support the presence of two distinct cleavage sites for the gene. (B) Density plot showing the distribution of lengths between proximal and distal cleavage sites for the genes that have more than one cleavage site. The median is 81 nucleotides. (C) Density plot showing the fold change in 3' UTR length between distal and proximal cleavage sites. Median is a 2.18-fold change. (D) Distribution of 3' UTR lengths for genes with a single cleavage site (left), the proximal sites for APA genes (middle), and the distal sites (right). (E) Poly(A) signal usage in APA genes. Sequences 30 nucleotides upstream of proximal and distal cleavage sites were used to search for the motifs described in Figure 2B. Plotted is the frequency of each motif across proximal (orange) and distal (red) cleavage sites.

\section{SUPPLEMENTAL FIGURE LEGENDS}

Supplemental figure 1. ONT sequencing allows for validation of cleavage sites. (A) Table showing the number of genes and sites for each step in our pipeline. "QuantSeq overlap" represents the $3^{\prime}$ UTRs that show at least $90 \%$ overlap between biological replicates. (B) Genome browser 
image looking at the 3' end of GL50803_21942 (NADP-specific glutamate dehydrogenase). 3'end libraries and ONT libraries both show a cleavage site 22 nucleotides downstream from the stop codon, which is consistent with previous observations.

Supplemental figure 2. Poly(A)-tail lengths in G. Iamblia. (A) Distribution of poly(A)-tail lengths in ONT libraries. Histogram shows the poly(A)-tail length of all reads that were assigned to a gene and for which a poly(A) tail was measured. Median is 62.1 nucleotides. (B) Hexagonal heatmap comparing poly(A)-tail measurements between ONT replicates. mRNAs with at least ten reads in both ONT datasets were selected and median poly(A)-tail lengths compared. (C-E) Genome browser images looking at GL50803_r0021 (28S ribosomal RNA), GL50803_r0019 (18S ribosomal RNA) and GL50803_r0020 (18S ribosomal RNA). Colored arrow illustrates the length and direction of the annotated gene. Grey arrows represent other genes present on the chromosome or contig being displayed. Shown are ONT reads on the positive (top) and negative (bottom) strands from two biological replicates.

Supplemental figure 3. Cleavage and polyadenylation contexts in humans. (A) Nucleotide frequency surrounding human cleavage sites. Sequences surrounding the end coordinates of all mRNAs in the RefSeq annotation were used to calculate nucleotide frequencies 30 nucleotides upstream and downstream of cleavage sites. (B) Distances between AAUAAA poly(A) signal and cleavage sites in human transcripts. mRNA sequences were downloaded from RefSeq annotations. All sequences with an AAUAAA in the last 40 nucleotides of the transcript were selected and the distance was calculated between the motif and the end of the mRNA. Distances are counted from the first A of the motif. 


\section{Supplemental figure 4. Gene differences between proximal and distal cleavage site usage. (A)}

The number of alternative polyadenylation events in G. lamblia is greater than previously thought. Bar plot shows the number of genes that were believed to contain more than one cleavage site based on previous literature, our 3'-end libraries alone (QuantSeq), and our validated list of cleavage sites (Nanopore). (B) Genome browser image showing a gene (GL50803_27887) with a short and long 3' UTR measuring 86 and 984 nucleotides, respectively. (C) FPKM expression for genes with a single cleavage site (left) or multiple cleavage sites (right). Median values are 86 and 59.2, respectively. (D) Distribution of poly(A)-tail lengths for genes with a single cleavage site (left) or multiple cleavage sites (right). Median values are 69 and 68.2 nucleotides, respectively.

Supplemental table 1. Validated 3' UTR sites in G. lamblia. Table of all validated 3' UTR lengths generated in this study. Genes are in separate sheets according to the number of cleavage sites.

Supplemental table 2. Poly(A)-tail lengths for G. Iamblia transcripts. Table of poly(A)-tail lengths for transcripts with at least ten reads in either ONT replicate. Listed are the number of reads in each replicate, median tail lengths and gene descriptions.

\section{Supplemental table 3. Predicted orthologs for cleavage and polyadenylation proteins in $G$.}

Iamblia. List of human cleavage and polyadenylation factors and putative G. lamblia orthologs. Human proteins were taken from (Tian \& Graber, 2012). Orthologs for G. lamblia were identified as described in methods. 


\section{REFERENCES}

Adiconis, X., Borges-Rivera, D., Satija, R., DeLuca, D. S., Busby, M. A., Berlin, A. M., Sivachenko, A., Thompson, D. A., Wysoker, A., Fennell, T., Gnirke, A., Pochet, N., Regev, A., \& Levin, J. Z. (2013). Comparative analysis of RNA sequencing methods for degraded or low-input samples. Nature Methods, 10(7), 623-629.

https://doi.org/10.1038/nmeth.2483

Ankarklev, J., Jerlström-Hultqvist, J., Ringqvist, E., Troell, K., \& Svärd, S. G. (2010). Behind the smile: Cell biology and disease mechanisms of Giardia species. Nature Reviews Microbiology, 8, 10.

Ashburner, M., Ball, C. A., Blake, J. A., Botstein, D., Butler, H., Cherry, J. M., Davis, A. P., Dolinski, K., Dwight, S. S., Eppig, J. T., Harris, M. A., Hill, D. P., Issel-Tarver, L., Kasarskis, A., Lewis, S., Matese, J. C., Richardson, J. E., Ringwald, M., Rubin, G. M., \& Sherlock, G. (2000). Gene Ontology: Tool for the unification of biology. Nature Genetics, 25(1), 25-29. https://doi.org/10.1038/75556

Baejen, C., Torkler, P., Gressel, S., Essig, K., Söding, J., \& Cramer, P. (2014). Transcriptome Maps of mRNP Biogenesis Factors Define Pre-mRNA Recognition. Molecular Cell, 55(5), 745-757. https://doi.org/10.1016/j.molcel.2014.08.005

Bailey, T. L., Boden, M., Buske, F. A., Frith, M., Grant, C. E., Clementi, L., Ren, J., Li, W. W., \& Noble, W. S. (2009). MEME SUITE: Tools for motif discovery and searching. Nucleic Acids Research, 37(Web Server), W202-W208. https://doi.org/10.1093/nar/gkp335

Barabino, S. M. L., Ohnacker, M., \& Keller, W. (2000). Distinct roles of two Yth1p domains in 3'-end cleavage and polyadenylation of yeast pre-mRNAs. The EMBO Journal, 19(14), 3778-3787. https://doi.org/10.1093/emboj/19.14.3778

Beaudoing, E. (2000). Patterns of Variant Polyadenylation Signal Usage in Human Genes. Genome Research, 10(7), 1001-1010. https://doi.org/10.1101/gr.10.7.1001

Best, A. A. (2004). Evolution of Eukaryotic Transcription: Insights From the Genome of Giardia lamblia. Genome Research, 14(8), 1537-1547. https://doi.org/10.1101/gr.2256604

Birse, C. E. (1997). Transcriptional termination signals for RNA polymerase II in fission yeast. The EMBO Journal, 16(12), 3633-3643. https://doi.org/10.1093/emboj/16.12.3633

Brown, K. M., \& Gilmartin, G. M. (2003). A Mechanism for the Regulation of Pre-mRNA 3' Processing by Human Cleavage Factor Im. Molecular Cell, 12(6), 1467-1476. https://doi.org/10.1016/S1097-2765(03)00453-2

Burki, F., Roger, A. J., Brown, M. W., \& Simpson, A. G. B. (2020). The New Tree of Eukaryotes. Trends in Ecology \& Evolution, 35(1), 43-55. https://doi.org/10.1016/j.tree.2019.08.008

Cacciò, S. M., \& Ryan, U. (2008). Molecular epidemiology of giardiasis. Molecular and Biochemical Parasitology, 160(2), 75-80. https://doi.org/10.1016/j.molbiopara.2008.04.006

Campbell, D. A., Thomas, S., \& Sturm, N. R. (2003). Transcription in kinetoplastid protozoa: Why be normal? Microbes and Infection, 5(13), 1231-1240. https://doi.org/10.1016/j.micinf.2003.09.005

Casañal, A., Kumar, A., Hill, C. H., Easter, A. D., Emsley, P., Degliesposti, G., Gordiyenko, Y., Santhanam, B., Wolf, J., Wiederhold, K., Dornan, G. L., Skehel, M., Robinson, C. V., \& Passmore, L. A. (2017). Architecture of eukaryotic mRNA 3'-end processing machinery. Science, 358(6366), 1056-1059. https://doi.org/10.1126/science.aao6535 
Chan, S., Choi, E.-A., \& Shi, Y. (2011). Pre-mRNA 3'-end processing complex assembly and function: Pre-mRNA 3'-end processing complex assembly. Wiley Interdisciplinary Reviews: RNA, 2(3), 321-335. https://doi.org/10.1002/wrna.54

Chan, S. L., Huppertz, I., Yao, C., Weng, L., Moresco, J. J., Yates, J. R., Ule, J., Manley, J. L., \& Shi, Y. (2014). CPSF30 and Wdr33 directly bind to AAUAAA in mammalian mRNA 3' processing. Genes \& Development, 28(21), 2370-2380. https://doi.org/10.1101/gad.250993.114

Chang, H., Lim, J., Ha, M., \& Kim, V. N. (2014). TAIL-seq: Genome-wide Determination of Poly(A) Tail Length and 3' End Modifications. Molecular Cell, 53(6), 1044-1052. https://doi.org/10.1016/j.molcel.2014.02.007

Clayton, C. (2013). The Regulation of Trypanosome Gene Expression by RNA-Binding Proteins. PLoS Pathogens, 9(11), e1003680. https://doi.org/10.1371/journal.ppat.1003680

Clayton, C. (2019). Regulation of gene expression in trypanosomatids: Living with polycistronic transcription. Open Biology, 9(6), 190072. https://doi.org/10.1098/rsob.190072

Clayton, C., \& Michaeli, S. (2011). 3' processing in protists. Wiley Interdisciplinary Reviews: RNA, 2(2), 247-255. https://doi.org/10.1002/wrna.49

Clerici, M., Faini, M., Muckenfuss, L. M., Aebersold, R., \& Jinek, M. (2018). Structural basis of AAUAAA polyadenylation signal recognition by the human CPSF complex. Nature Structural \& Molecular Biology, 25(2), 135-138. https://doi.org/10.1038/s41594-0170020-6

Collart, M. A., \& Oliviero, S. (1993). Preparation of Yeast RNA. Current Protocols in Molecular Biology, 23, 12-13.

Dichtl, B. (2002). Yhh1p/Cft1p directly links poly(A) site recognition and RNA polymerase II transcription termination. The EMBO Journal, 21(15), 4125-4135. https://doi.org/10.1093/emboj/cdf390

Dobin, A., Davis, C. A., Schlesinger, F., Drenkow, J., Zaleski, C., Jha, S., Batut, P., Chaisson, M., \& Gingeras, T. R. (2013). STAR: ultrafast universal RNA-seq aligner. Bioinformatics, 29(1), 7.

Eiler, D. R., Wimberly, B. T., Bilodeau, D. Y., Rissland, O. S., \& Kieft, J. S. (2020). The Giardia lamblia ribosome structure reveals divergence in translation and quality control pathways. BioRxiv. https://doi.org/10.1101/2020.09.30.321331

Einarsson, E., Troell, K., Hoeppner, M. P., Grabherr, M., Ribacke, U., \& Svärd, S. G. (2016). Coordinated Changes in Gene Expression Throughout Encystation of Giardia intestinalis. PLOS Neglected Tropical Diseases, 10(3), e0004571. https://doi.org/10.1371/journal.pntd.0004571

Franzén, O., Jerlström-Hultqvist, J., Einarsson, E., Ankarklev, J., Ferella, M., Andersson, B., \& Svärd, S. G. (2013). Transcriptome Profiling of Giardia intestinalis Using Strand-specific RNA-Seq. PLOS Computational Biology, 9(3), e1003000. https://doi.org/10.1371/journal.pcbi.1003000

Gallie, D. R. (1991). The cap and poly(A) tail function synergistically to regulate mRNA translational efficiency. Genes \& Development, 5(11), 2108-2116. https://doi.org/10.1101/gad.5.11.2108

Gross, S., \& Moore, C. L. (2001). Rna15 Interaction with the A-Rich Yeast Polyadenylation Signal Is an Essential Step in mRNA 3J-End Formation. MOL. CELL. BIOL., 21, 11. 
Hehl, A. B., Marti, M., \& Köhler, P. (2000). Stage-Specific Expression and Targeting of Cyst Wall Protein-Green Fluorescent Protein Chimeras in Giardia. Molecular Biology of the Cell, 11(5), 1789-1800. https://doi.org/10.1091/mbc.11.5.1789

Hendriks, E. F., Abdul-Razak, A., \& Matthews, K. R. (2003). TbCPSF30 Depletion by RNA Interference Disrupts Polycistronic RNA Processing in Trypanosoma brucei. Journal of Biological Chemistry, 278(29), 26870-26878. https://doi.org/10.1074/jbc.M302405200

Hill, C. H., Boreikaite, V., Kumar, A., Casañal, A., Kubík, P., Degliesposti, G., Maslen, S., Mariani, A., von Loeffelholz, O., Girbig, M., Skehel, M., \& Passmore, L. A. (2019). Activation of the Endonuclease that Defines mRNA 3' Ends Requires Incorporation into an 8-Subunit Core Cleavage and Polyadenylation Factor Complex. Molecular Cell, 73(6), 1217-1231.e11. https://doi.org/10.1016/j.molcel.2018.12.023

Hon, C.-C., Weber, C., Sismeiro, O., Proux, C., Koutero, M., Deloger, M., Das, S., Agrahari, M., Dillies, M.-A., Jagla, B., Coppee, J.-Y., Bhattacharya, A., \& Guillen, N. (2013). Quantification of stochastic noise of splicing and polyadenylation in Entamoeba histolytica. Nucleic Acids Research, 41(3), 1936-1952. https://doi.org/10.1093/nar/gks1271

Hoque, M., Ji, Z., Zheng, D., Luo, W., Li, W., You, B., Park, J. Y., Yehia, G., \& Tian, B. (2013). Analysis of alternative cleavage and polyadenylation by $3^{\prime}$ region extraction and deep sequencing. Nature Methods, 10(2), 133-139. https://doi.org/10.1038/nmeth.2288

Hu, J., Lutz, C. S., Wilusz, J., \& Tian, B. (2005). Bioinformatic identification of candidate cisregulatory elements involved in human mRNA polyadenylation. $R N A, 11(10), 1485-$ 1493. https://doi.org/10.1261/rna.2107305

Iyer, V., Chettiar, S. T., Grover, M., Rajyaguru, P., Nageshan, R. K., \& Tatu, U. (2019). Giardia lamblia Hsp90 pre-mRNAs undergo self-splicing to generate mature RNA in an in vitro trans-splicing reaction. FEBS Letters, 593(4), 433-442. https://doi.org/10.1002/18733468.13324

Keister, D. B. (1983). Axenic culture of Giardia lamblia in TYI-S-33 medium supplemented with bile. Transactions of the Royal Society of Tropical Medicine and Hygiene, 77(4), 487488. https://doi.org/10.1016/0035-9203(83)90120-7

Koch, H., Raabe, M., Urlaub, H., Bindereif, A., \& Preußer, C. (2016). The polyadenylation complex of Trypanosoma brucei: Characterization of the functional poly(A) polymerase. RNA Biology, 13(2), 221-231. https://doi.org/10.1080/15476286.2015.1130208

Krause, M., Niazi, A. M., Labun, K., Cleuren, Y. N. T., Müller, F. S., \& Valen, E. (2019). tailfindr: Alignment-free poly(A) length measurement for Oxford Nanopore RNA and DNA sequencing. $R N A, 25,13$.

Kumar, A., Clerici, M., Muckenfuss, L. M., Passmore, L. A., \& Jinek, M. (2019). Mechanistic insights into mRNA 3'-end processing. Current Opinion in Structural Biology, 59, 143150. https://doi.org/10.1016/j.sbi.2019.08.001

LaCava, J., Houseley, J., Saveanu, C., Petfalski, E., Thompson, E., Jacquier, A., \& Tollervey, D. (2005). RNA Degradation by the Exosome Is Promoted by a Nuclear Polyadenylation Complex. Cell, 121(5), 713-724. https://doi.org/10.1016/j.cell.2005.04.029

Legendre, M., \& Gautheret, D. (2003). Sequence determinants in human polyadenylation site selection. BMC Genomics, 4(1), 7. https://doi.org/10.1186/1471-2164-4-7

Li, H. (2018). Minimap2: Pairwise alignment for nucleotide sequences. Bioinformatics, 34(18), 7. 
Li, L., \& Wang, C. C. (2004). Capped mRNA with a Single Nucleotide Leader Is Optimally Translated in a Primitive Eukaryote, Giardia lamblia. Journal of Biological Chemistry, 279(15), 14656-14664. https://doi.org/10.1074/jbc.M309879200

Li, X.-Q., \& Du, D. (2014). Motif types, motif locations and base composition patterns around the RNA polyadenylation site in microorganisms, plants and animals. BMC Evolutionary Biology, 14(1), 162. https://doi.org/10.1186/s12862-014-0162-7

Liao, Y., Smyth, G. K., \& Shi, W. (2014). featureCounts: An efficient general purpose program for assigning sequence reads to genomic features. Bioinformatics, 30(7), 923-930. https://doi.org/10.1093/bioinformatics/btt656

Lima, S. A., Chipman, L. B., Nicholson, A. L., Chen, Y.-H., Yee, B. A., Yeo, G. W., Coller, J., \& Pasquinelli, A. E. (2017). Short poly(A) tails are a conserved feature of highly expressed genes. Nature Structural \& Molecular Biology, 24(12), 1057-1063. https://doi.org/10.1038/nsmb.3499

Liu, X., Freitas, J., Zheng, D., Oliveira, M. S., Hoque, M., Martins, T., Henriques, T., Tian, B., \& Moreira, A. (2017). Transcription elongation rate has a tissue-specific impact on alternative cleavage and polyadenylation in Drosophila melanogaster. $R N A, 23(12)$, 1807-1816. https://doi.org/10.1261/rna.062661.117

Loman, N. J., Quick, J., \& Simpson, J. T. (2015). A complete bacterial genome assembled de novo using only nanopore sequencing data. Nature Methods, 12(8), 733-735. https://doi.org/10.1038/nmeth.3444

Lu, Y., Gao, C., \& Han, B. (2006). Sequence analysis of mRNA polyadenylation signals of rice genes. Chinese Science Bulletin, 51(9), 1069-1077. https://doi.org/10.1007/s11434-0061069-5

Mandel, C. R., Kaneko, S., Zhang, H., Gebauer, D., Vethantham, V., Manley, J. L., \& Tong, L. (2006). Polyadenylation factor CPSF-73 is the pre-mRNA 3'-end-processing endonuclease. Nature, 444(7121), 953-956. https://doi.org/10.1038/nature05363

Mayr, C. (2017). Regulation by 3'-Untranslated Regions. Annual Review of Genetics, 51(1), 171-194. https://doi.org/10.1146/annurev-genet-120116-024704

Mayr, C., \& Bartel, D. P. (2009). Widespread Shortening of 3'UTRs by Alternative Cleavage and Polyadenylation Activates Oncogenes in Cancer Cells. Cell, 138(4), 673-684. https://doi.org/10.1016/j.cell.2009.06.016

Meyer, A. (1976). Giardia lamblia: Isolation and Axenic Cultivation. Experimental Parasitology, $39,101-105$.

Millevoi, S., \& Vagner, S. (2010). Molecular mechanisms of eukaryotic pre-mRNA 3' end processing regulation. Nucleic Acids Research, 38(9), 2757-2774. https://doi.org/10.1093/nar/gkp1176

Mok, M. T. S., Tay, E., Sekyere, E., Glenn, W. K., Bagnara, A. S., \& Edwards, M. R. (2005). Giardia intestinalis: Molecular characterization of UDP-N-acetylglucosamine pyrophosphorylase. Gene, 357(1), 73-82. https://doi.org/10.1016/j.gene.2005.05.010

Monis, P. T., Caccio, S. M., \& Thompson, R. C. A. (2009). Variation in Giardia: Towards a taxonomic revision of the genus. Trends in Parasitology, 25(2), 93-100. https://doi.org/10.1016/j.pt.2008.11.006

Moqtaderi, Z., Geisberg, J. V., \& Struhl, K. (2018). Extensive Structural Differences of Closely Related 3' mRNA Isoforms: Links to Pab1 Binding and mRNA Stability. Molecular Cell, 72(5), 849-861.e6. https://doi.org/10.1016/j.molcel.2018.08.044 
Morris, A. R., Bos, A., Diosdado, B., Rooijers, K., Elkon, R., Bolijn, A. S., Carvalho, B., Meijer, G. A., \& Agami, R. (2012). Alternative Cleavage and Polyadenylation during Colorectal Cancer Development. Clinical Cancer Research, 18(19), 5256-5266. https://doi.org/10.1158/1078-0432.CCR-12-0543

Morrison, H. G., McArthur, A. G., Gillin, F. D., Aley, S. B., Adam, R. D., Olsen, G. J., Best, A. A., Cande, W. Z., Chen, F., Cipriano, M. J., Davids, B. J., Dawson, S. C., Elmendorf, H. G., Hehl, A. B., Holder, M. E., Huse, S. M., Kim, U. U., Lasek-Nesselquist, E., Manning, G., ... Sogin, M. L. (2007). Genomic Minimalism in the Early Diverging Intestinal Parasite Giardia lamblia. Science, 317(5846), 1921-1926. https://doi.org/10.1126/science.1143837

Nam, D. K., Lee, S., Zhou, G., Cao, X., Wang, C., Clark, T., Chen, J., Rowley, J. D., \& Wang, S. M. (2002). Oligo(dT) primer generates a high frequency of truncated cDNAs through internal poly(A) priming during reverse transcription. Proceedings of the National Academy of Sciences, 99(9), 6152-6156. https://doi.org/10.1073/pnas.092140899

Nixon, J. E. J., Wang, A., Morrison, H. G., McArthur, A. G., Sogin, M. L., Loftus, B. J., \& Samuelson, J. (2002). A spliceosomal intron in Giardia lamblia. Proceedings of the National Academy of Sciences, 99(6), 3701-3705. https://doi.org/10.1073/pnas.042700299

Nourse, J., Spada, S., \& Danckwardt, S. (2020). Emerging Roles of RNA 3'-end Cleavage and Polyadenylation in Pathogenesis, Diagnosis and Therapy of Human Disorders. Biomolecules, 10(6), 915. https://doi.org/10.3390/biom10060915

Oguariri, R. M., Dunn, J. M., \& Golightly, L. M. (2006). 3' gene regulatory elements required for expression of the Plasmodium falciparum developmental protein, Pfs25. Molecular and Biochemical Parasitology, 146(2), 163-172. https://doi.org/10.1016/j.molbiopara.2005.12.004

Peattie, D. A., Alonso, R. A., Hein, A., \& Caulfield, J. P. (1989). Ultrastructural localization of giardins to the edges of disk microribbons of Giarida lamblia and the nucleotide and deduced protein sequence of alpha giardin. The Journal of Cell Biology, 109(5), 23232335. https://doi.org/10.1083/jcb.109.5.2323

Proudfoot, N. J., \& Brownlee, G. G. (1976). 3' Non-coding region sequences in eukaryotic messenger RNA. Nature, 263(5574), 211-214. https://doi.org/10.1038/263211a0

Que, X., Svärd, S. G., Meneb, T.-C., Hetsko, M. L., Aley, S. B., \& Gillin, F. D. (1996). Developmentally regulated transcripts and evidence of differential mRNA processing in Giardia lamblia. Molecular and Biochemical Parasitology, 81, 10.

Rissland, O. S. (2017). The organization and regulation of mRNA-protein complexes: MRNP organization and regulation. Wiley Interdisciplinary Reviews: RNA, 8(1), e1369. https://doi.org/10.1002/wrna.1369

Sandberg, R., Neilson, J. R., Sarma, A., Sharp, P. A., \& Burge, C. B. (2008). Proliferating Cells Express mRNAs with Shortened 3' Untranslated Regions and Fewer MicroRNA Target Sites. Science, 320(5883), 1643-1647. https://doi.org/10.1126/science.1155390

Schönemann, L., Kühn, U., Martin, G., Schäfer, P., Gruber, A. R., Keller, W., Zavolan, M., \& Wahle, E. (2014). Reconstitution of CPSF active in polyadenylation: Recognition of the polyadenylation signal by WDR33. Genes \& Development, 28(21), 2381-2393. https://doi.org/10.1101/gad.250985.114

Sheets, M. D., Ogg, S. C., \& Wickens, M. P. (1990). Point mutations in AAUAAA and the poly (A) addition site: Effects on the accuracy and efficiency of cleavage and polyadenylation 
in vitro. Nucleic Acids Research, 18(19), 5799-5805.

https://doi.org/10.1093/nar/18.19.5799

Siegel, T., Hon, C.-C., Zhang, Q., Lopez-Rubio, J.-J., Scheidig-Benatar, C., Martins, R. M., Sismeiro, O., Coppée, J.-Y., \& Scherf, A. (2014). Strand-specific RNA-Seq reveals widespread and developmentally regulated transcription of natural antisense transcripts in Plasmodium falciparum. BMC Genomics, 15(1), 150. https://doi.org/10.1186/1471-2164$15-150$

Singh, G., Pratt, G., Yeo, G. W., \& Moore, M. J. (2015). The Clothes Make the mRNA: Past and Present Trends in mRNP Fashion. Annual Review of Biochemistry, 84(1), 325-354. https://doi.org/10.1146/annurev-biochem-080111-092106

Stevens, A. T., Howe, D. K., \& Hunt, A. G. (2018). Characterization of mRNA polyadenylation in the apicomplexa. PLOS ONE, 13(8), e0203317. https://doi.org/10.1371/journal.pone.0203317

Subtelny, A. O., Eichhorn, S. W., Chen, G. R., Sive, H., \& Bartel, D. P. (2014). Poly(A)-tail profiling reveals an embryonic switch in translational control. Nature, 508(7494), 66-71. https://doi.org/10.1038/nature13007

Sullivan, K. D., Steiniger, M., \& Marzluff, W. F. (2009). A Core Complex of CPSF73, CPSF100, and Symplekin May Form Two Different Cleavage Factors for Processing of Poly(A) and Histone mRNAs. Molecular Cell, 34(3), 322-332. https://doi.org/10.1016/j.molcel.2009.04.024

Sun, Y., Zhang, Y., Hamilton, K., Manley, J. L., Shi, Y., Walz, T., \& Tong, L. (2018). Molecular basis for the recognition of the human AAUAAA polyadenylation signal. Proceedings of the National Academy of Sciences, 115(7), E1419-E1428. https://doi.org/10.1073/pnas.1718723115

Takagaki, Y., \& Manley, J. L. (1997). RNA recognition by the human polyadenylation factor CstF. Molecular and Cellular Biology, 17(7), 3907-3914. https://doi.org/10.1128/MCB.17.7.3907

The Gene Ontology Consortium, Carbon, S., Douglass, E., Good, B. M., Unni, D. R., Harris, N. L., Mungall, C. J., Basu, S., Chisholm, R. L., Dodson, R. J., Hartline, E., Fey, P., Thomas, P. D., Albou, L.-P., Ebert, D., Kesling, M. J., Mi, H., Muruganujan, A., Huang, X., ... Elser, J. (2021). The Gene Ontology resource: Enriching a GOld mine. Nucleic Acids Research, 49(D1), D325-D334. https://doi.org/10.1093/nar/gkaa1113

Tian, B., \& Graber, J. H. (2012). Signals for pre-mRNA cleavage and polyadenylation. Wiley Interdisciplinary Reviews: RNA, 3(3), 385-396. https://doi.org/10.1002/wrna.116

Tian, B., Hu, J., Zhang, H., \& Lutz, C. S. (2005). A large-scale analysis of mRNA polyadenylation of human and mouse genes. Nucleic Acids Research, 33(1), 201-212. https://doi.org/10.1093/nar/gki158

Venkataraman, K. (2005). Analysis of a noncanonical poly(A) site reveals a tripartite mechanism for vertebrate poly(A) site recognition. Genes \& Development, 19(11), 1315-1327. https://doi.org/10.1101/gad.1298605

Whitelaw, E., \& Proudfoot, N. (1986). $\alpha$-Thalassaemia caused by a poly(A) site mutation reveals that transcriptional termination is linked to 3' end processing in the human a2 globin gene. 8.

Workman, R. E., Tang, A. D., Tang, P. S., Jain, M., Tyson, J. R., Razaghi, R., Zuzarte, P. C., Gilpatrick, T., Payne, A., Quick, J., Sadowski, N., Holmes, N., de Jesus, J. G., Jones, K. L., Soulette, C. M., Snutch, T. P., Loman, N., Paten, B., Loose, M., ... Timp, W. (2019). 
Nanopore native RNA sequencing of a human poly(A) transcriptome. Nature Methods, 16(12), 1297-1305. https://doi.org/10.1038/s41592-019-0617-2

Xing, D., \& Li, Q. Q. (2011). Alternative polyadenylation and gene expression regulation in plants: Polyadenylation and gene expression regulation. Wiley Interdisciplinary Reviews: RNA, 2(3), 445-458. https://doi.org/10.1002/wrna.59

$\mathrm{Xu}, \mathrm{F}$., Jerlström-Hultqvist, J., Einarsson, E., Ástvaldsson, Á., Svärd, S. G., \& Andersson, J. O. (2014). The Genome of Spironucleus salmonicida Highlights a Fish Pathogen Adapted to Fluctuating Environments. PLoS Genetics, 10(2), e1004053. https://doi.org/10.1371/journal.pgen.1004053

Xu, F., Jex, A., \& Svärd, S. G. (2020). A chromosome-scale reference genome for Giardia intestinalis WB. Scientific Data, 7(1), 38. https://doi.org/10.1038/s41597-020-0377-y

Xu, F., Jiménez-González, A., Einarsson, E., Ástvaldsson, Á., Peirasmaki, D., Eckmann, L., Andersson, J. O., Svärd, S. G., \& Jerlström-Hultqvist, J. (2020). The compact genome of Giardia muris reveals important steps in the evolution of intestinal protozoan parasites. Microbial Genomics, 6(8). https://doi.org/10.1099/mgen.0.000402

Yee, J., \& Dennis, P. P. (1992). Isolation and Characterizationof a NADP-dependent Glutamate Dehydrogenase Gene from the Primitive Eucaryote Giardia lamblia. The Journal of Biological Chemistry, 267(11), 6.

Yu, F., Zhang, Y., Cheng, C., Wang, W., Zhou, Z., Rang, W., Yu, H., Wei, Y., Wu, Q., \& Zhang, Y. (2020). Poly(A)-seq: A method for direct sequencing and analysis of the transcriptomic poly(A)-tails. PLOS ONE, 15(6), e0234696.

https://doi.org/10.1371/journal.pone.0234696 

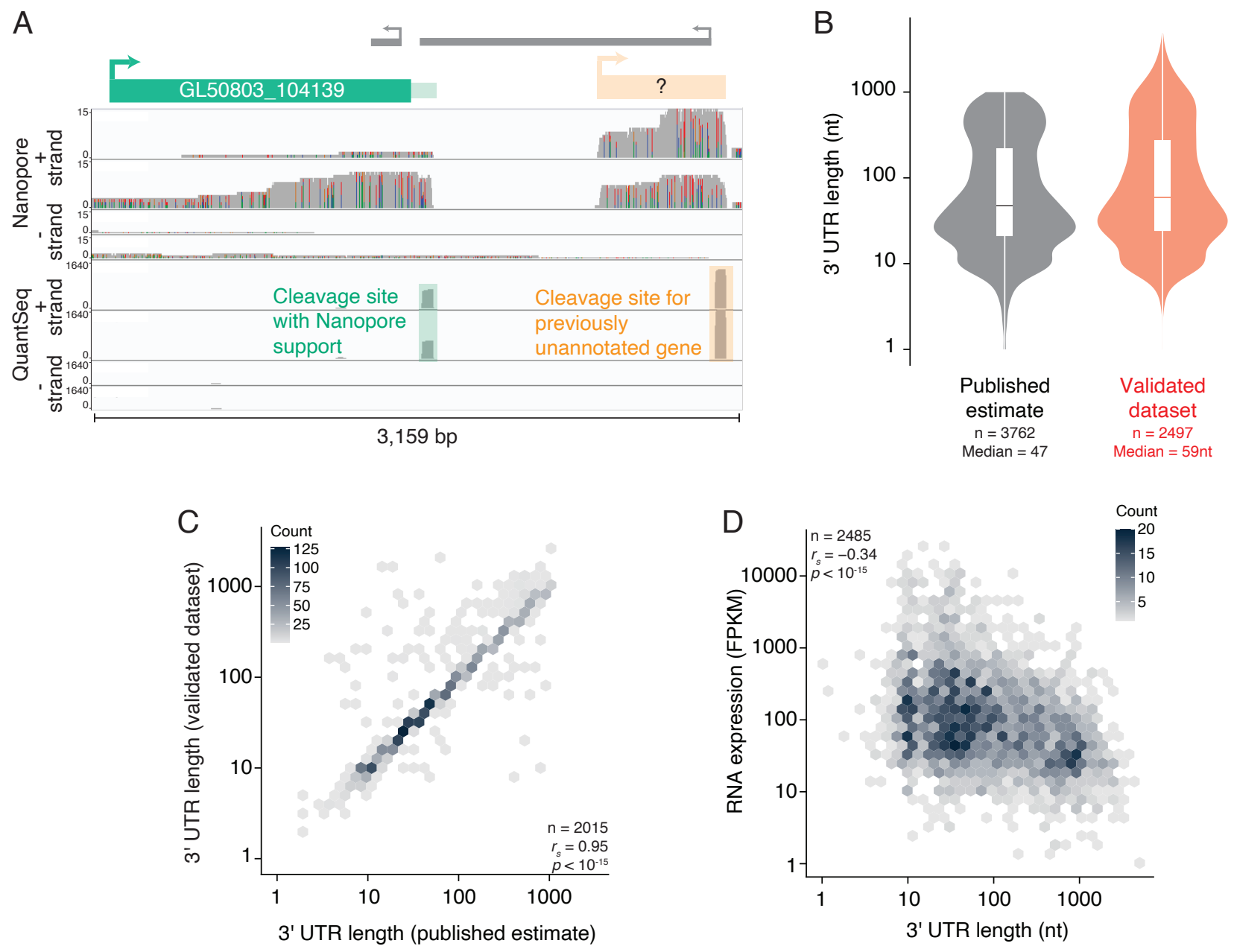

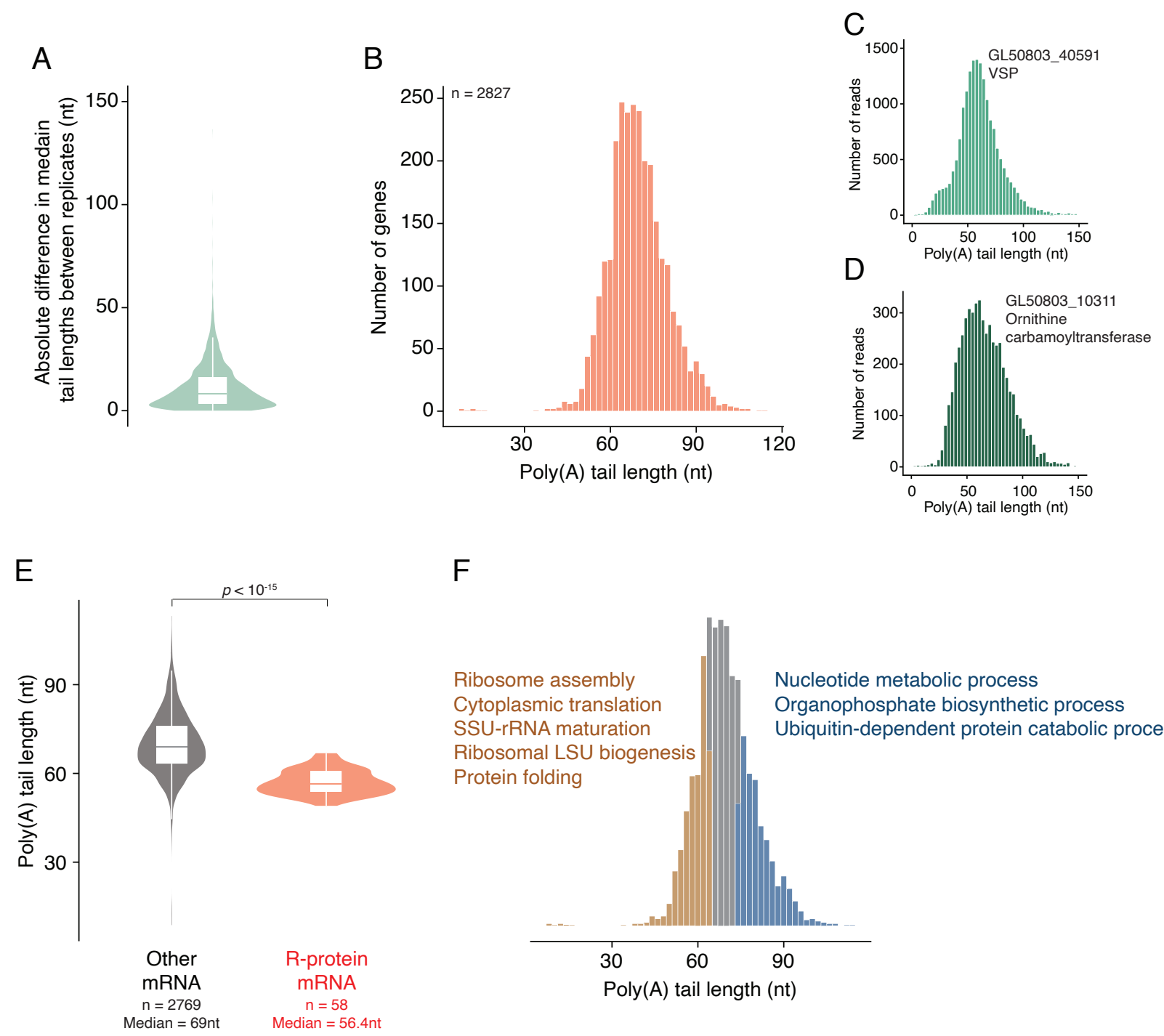

F

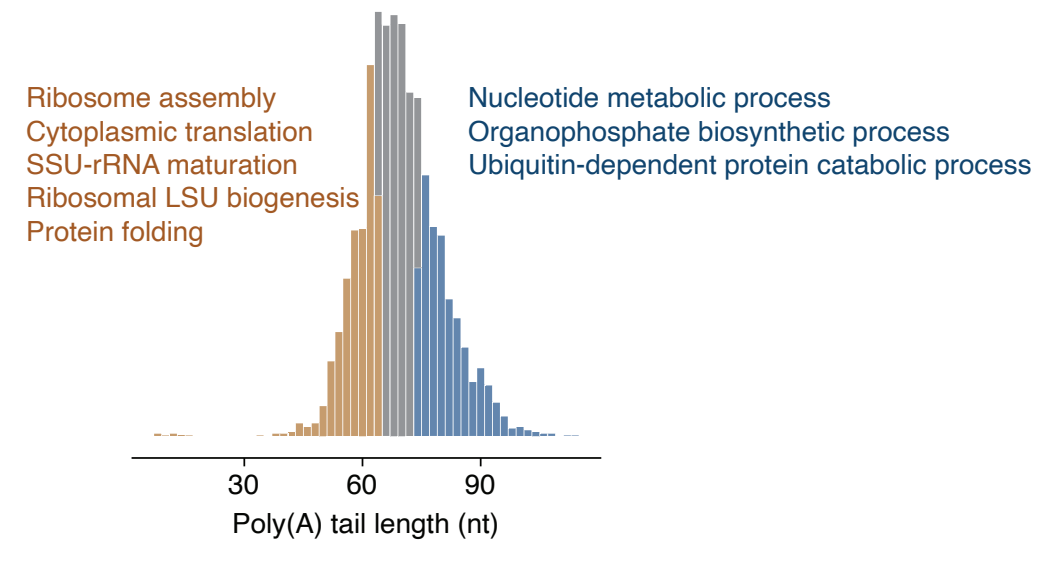




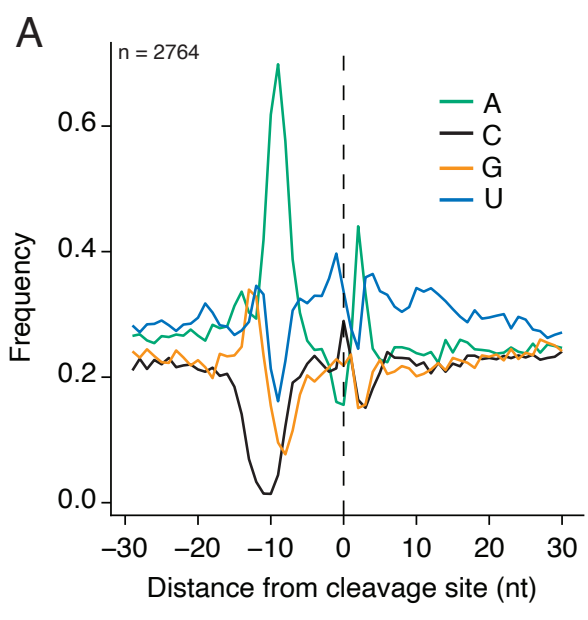

C

Human

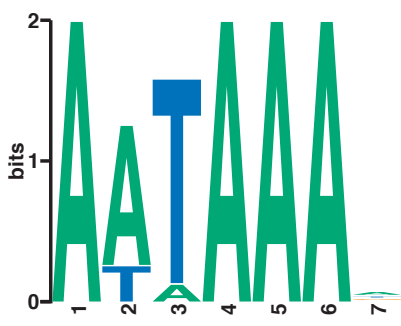

B

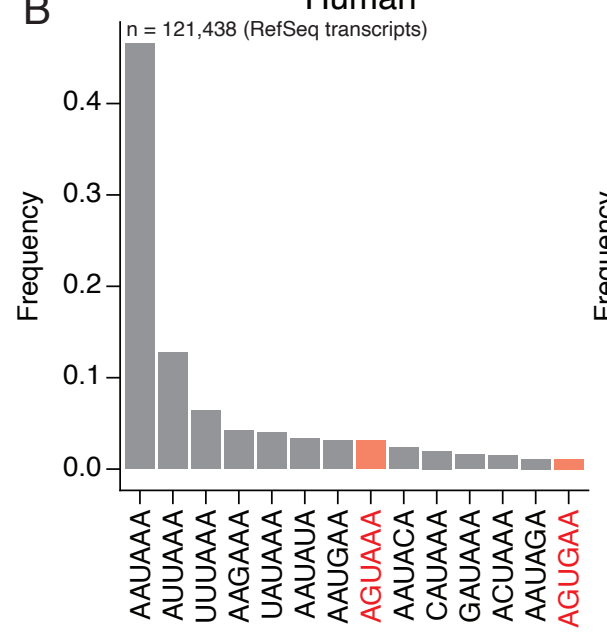

G. lamblia
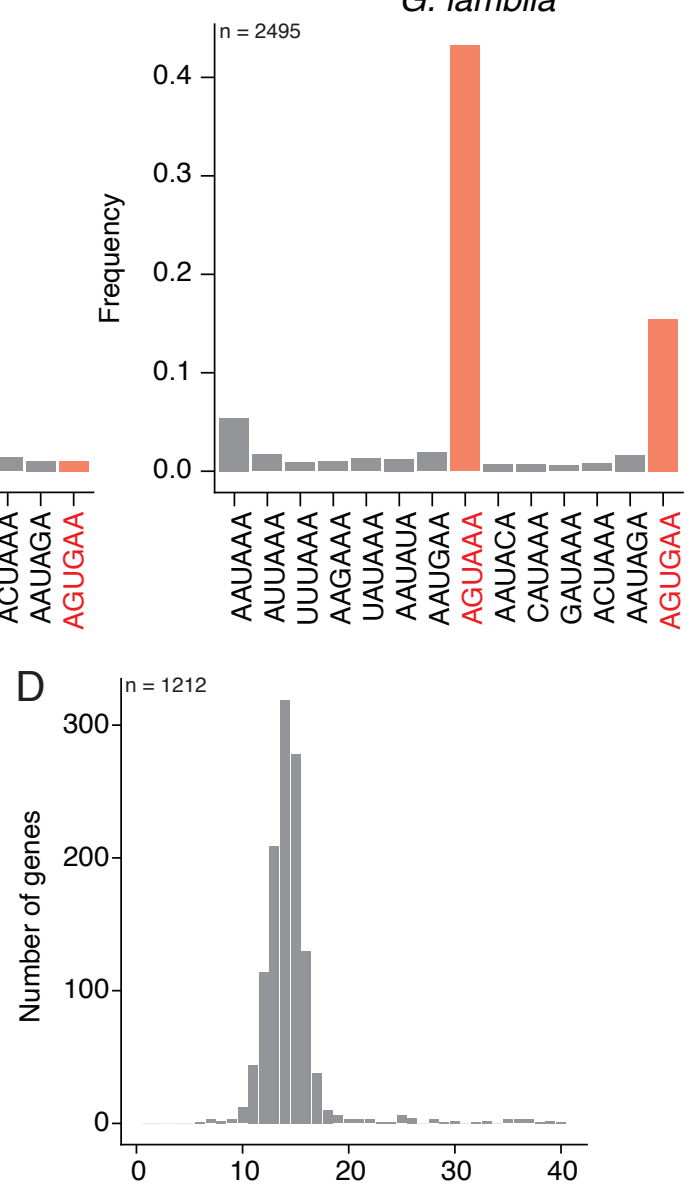

Distance between AGUAAA and cleavage site
G. Iamblia

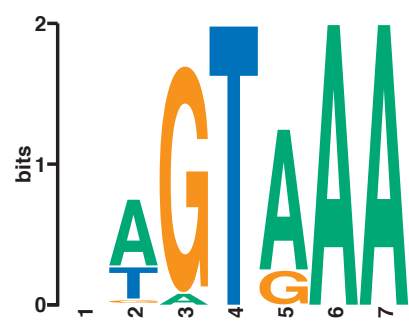


A

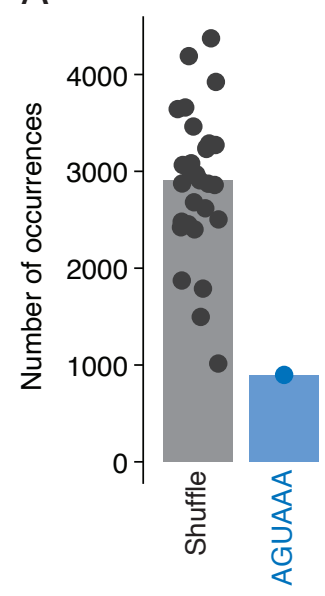

B

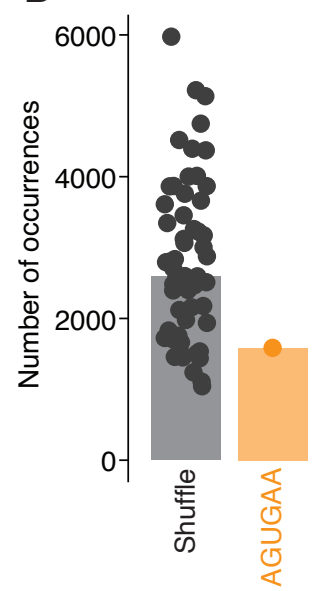

E

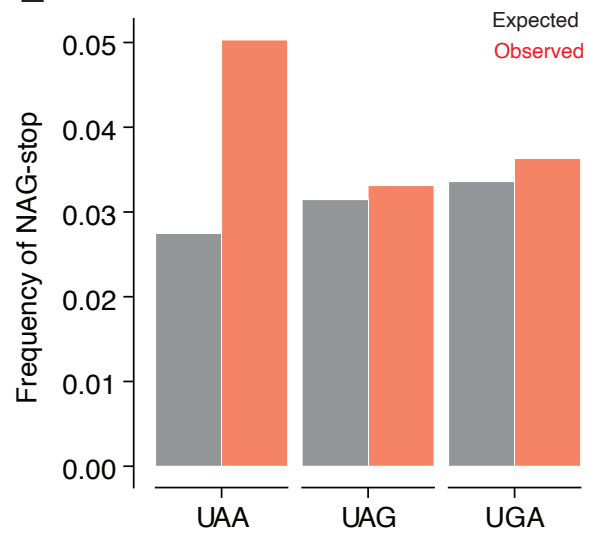

C

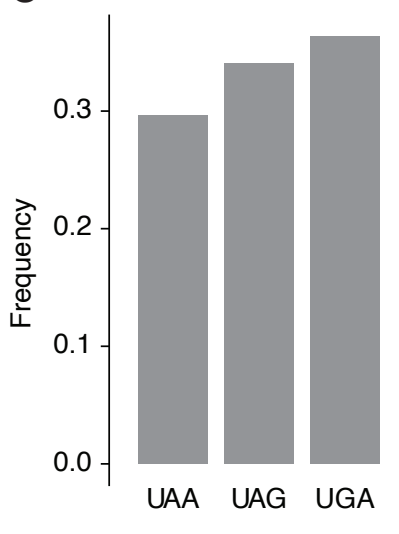

D

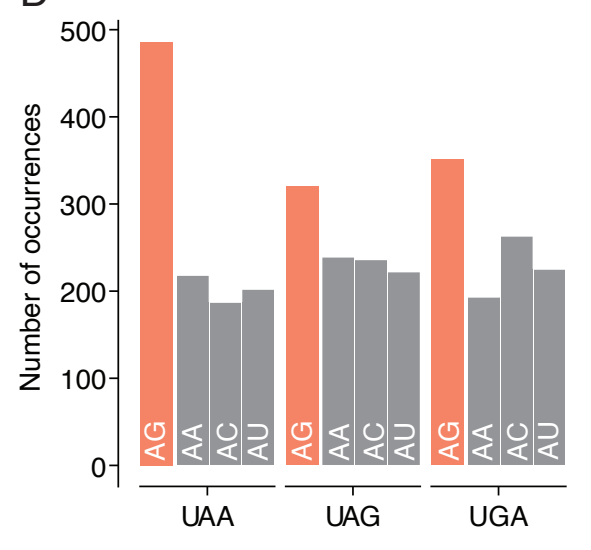

F

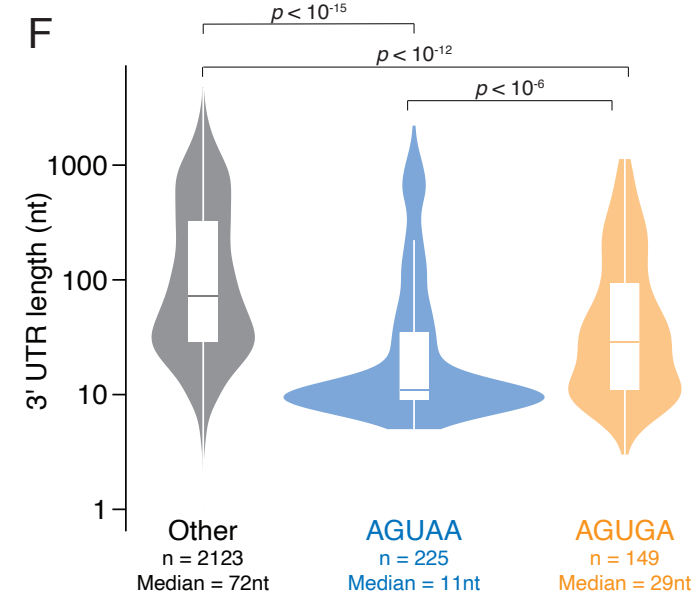


A

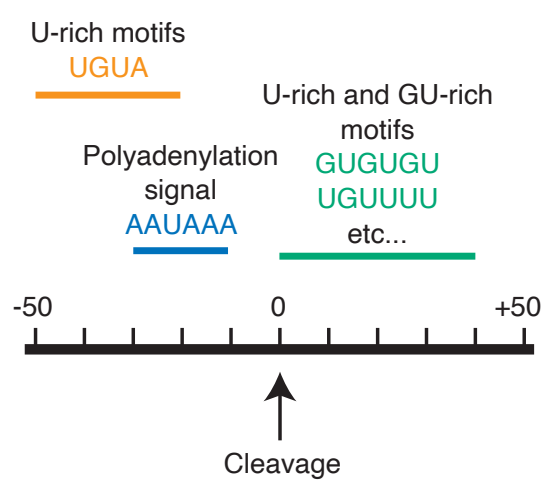

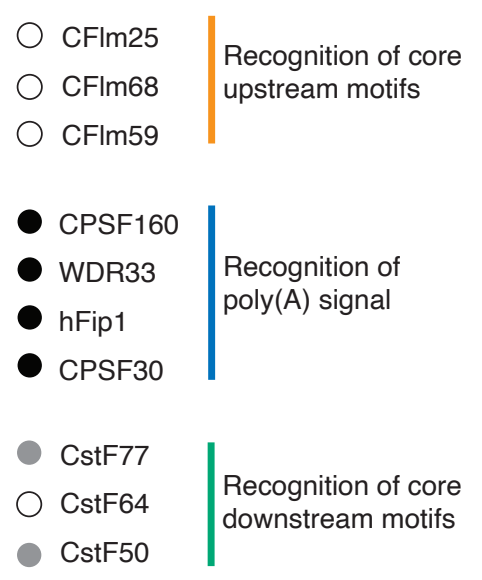

C
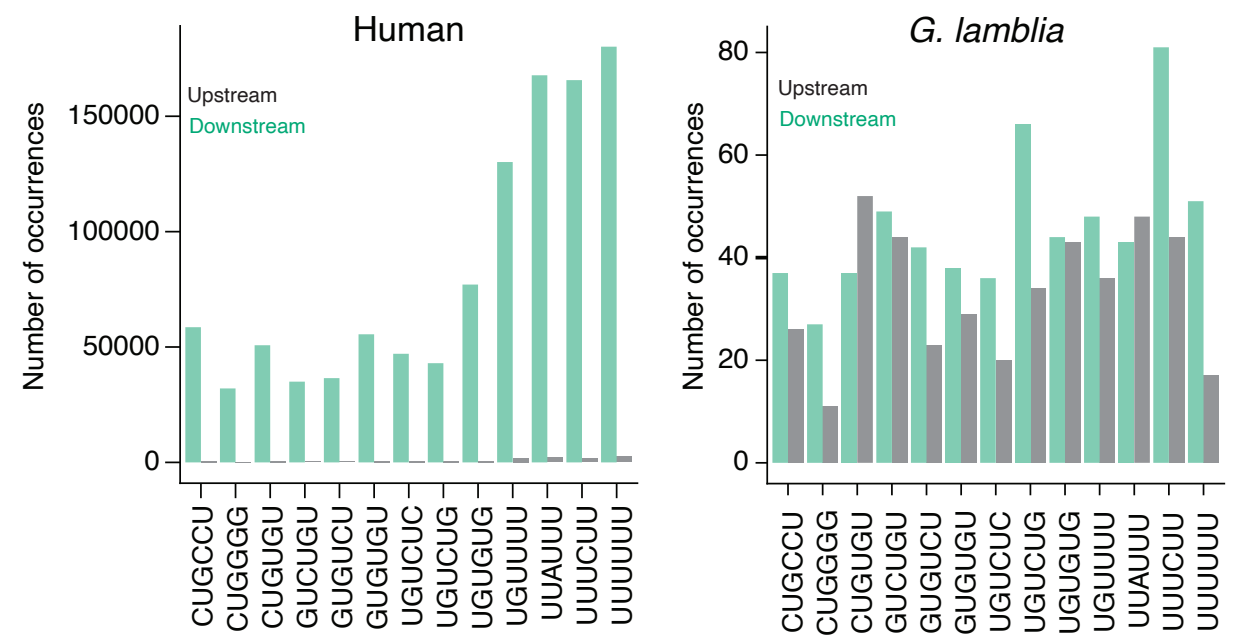

B

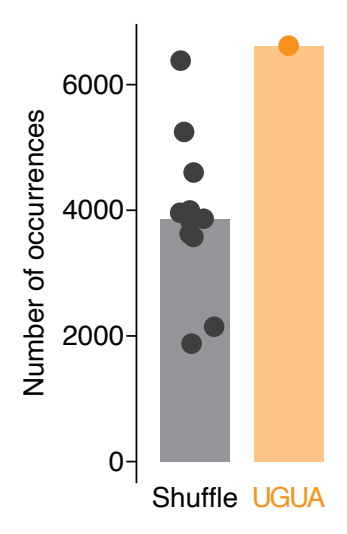

G. lamblia

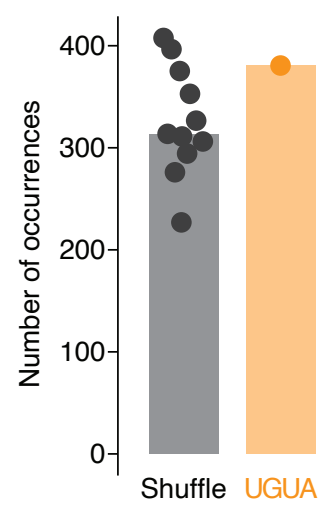

D

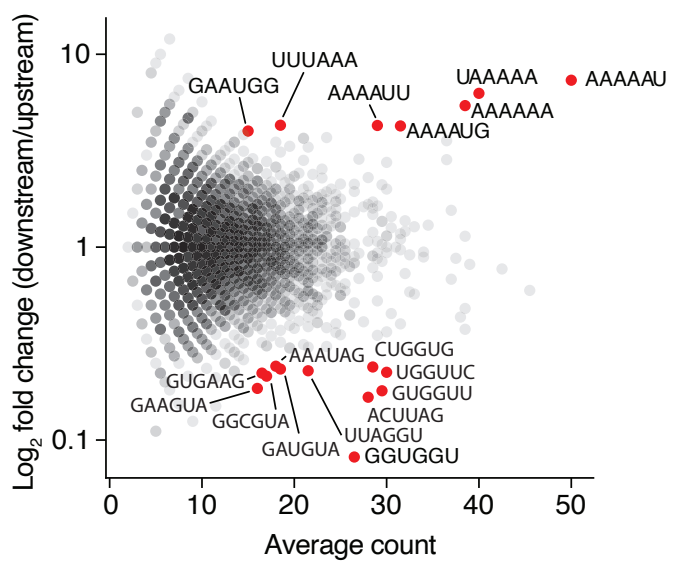



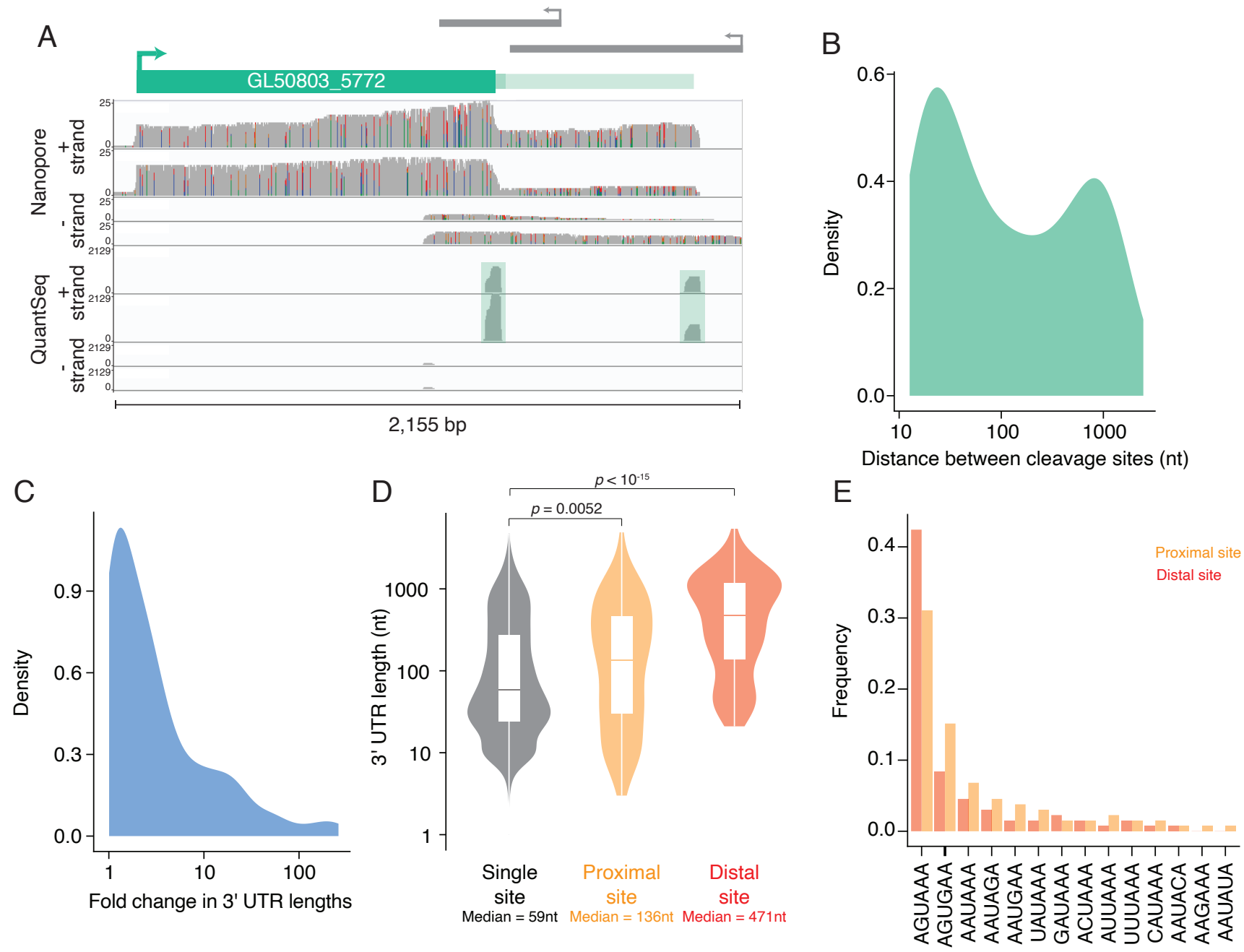

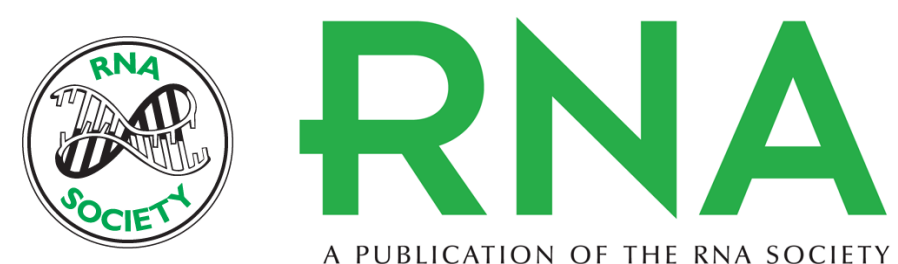

A PUBLICATION OF THE RNA SOCIETY

\title{
Precise gene models using long-read sequencing reveal a unique poly(A) signal in Giardia lamblia
}

\author{
Danielle Y Bilodeau, Ryan M Sheridan, Balu Balan, et al.
}

RNA published online February 2, 2022

\section{Supplemental} Material

$\mathbf{P}<\mathbf{P} \quad$ Published online February 2, 2022 in advance of the print journal.

Accepted Manuscript

Open Access

Creative Commons License

Email Alerting Service
http://rnajournal.cshlp.org/content/suppl/2022/02/02/rna.078793.121.DC1

Peer-reviewed and accepted for publication but not copyedited or typeset; accepted manuscript is likely to differ from the final, published version.

Freely available online through the RNA Open Access option.

This article, published in $R N A$, is available under a Creative Commons License (Attribution 4.0 International), as described at http://creativecommons.org/licenses/by/4.0/.

Receive free email alerts when new articles cite this article - sign up in the box at the top right corner of the article or click here.

Advance online articles have been peer reviewed and accepted for publication but have not yet appeared in the paper journal (edited, typeset versions may be posted when available prior to final publication). Advance online articles are citable and establish publication priority; they are indexed by PubMed from initial publication. Citations to Advance online articles must include the digital object identifier (DOIs) and date of initial publication.

To subscribe to RNA go to:

http://rnajournal.cshlp.org/subscriptions 